\title{
Time Resolved Stereo Particle Image Velocimetry Measurements of the Instabilities Downstream of a Backward-Facing Step in a Swept-Wing Boundary Layer
}

\author{
Jenna L. Eppink* and Chung-Sheng Yao* \\ NASA Langley Research Center, Hampton, VA 23681
}

\begin{abstract}
Time-resolved particle image velocimetry (TRPIV) measurements are performed downstream of a swept backward-facing step, with a height of $49 \%$ of the boundary-layer thickness. The results agree well qualitatively with previously reported hotwire measurements, though the amplitudes of the fluctuating components measured using TRPIV are higher. Nonetheless, the low-amplitude instabilities in the flow are fairly well resolved using TRPIV. Proper orthogonal decomposition is used to study the development of the traveling crossflow and Tollmien-Schlichting (TS) instabilities downstream of the step and to study how they interact to form the large velocity spikes that ultimately lead to transition. A secondary mode within the traveling crossflow frequency band develops with a wavelength close to that of the stationary crossflow instability, so that at a certain point in the phase, it causes an increase in the spanwise modulation initially caused by the stationary crossflow mode. This increased modulation leads to an increase in the amplitude of the TS mode, which, itself, is highly modulated through interactions with the stationary crossflow. When the traveling crossflow and TS modes align in time and space, the large velocity spikes occur. Thus, these three instabilities, which are individually of low amplitude when the spikes start to occur $\left(u_{r m s}^{\prime} / U_{e}<0.03\right)$, interact and combine to cause a large flow disturbance that eventually leads to transition.
\end{abstract}

\section{Nomenclature}

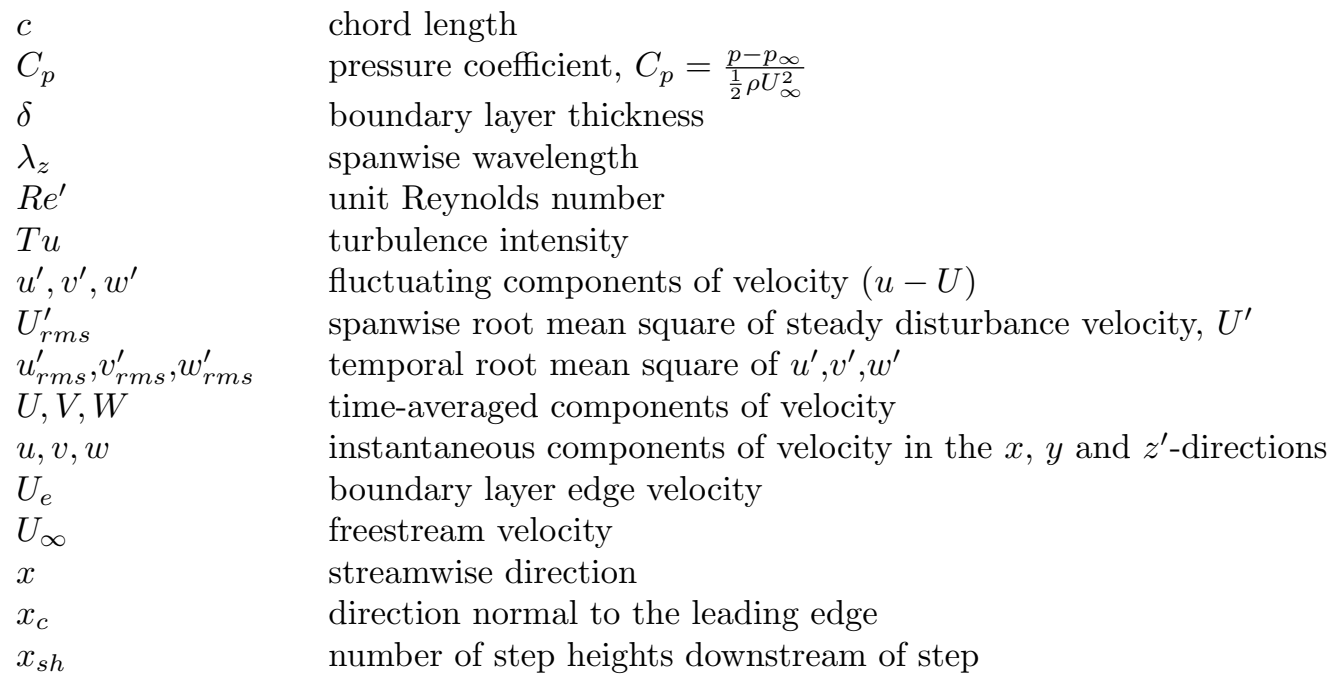

*Research Aerospace Engineer, Flow Physics and Control Branch, M.S. 170, AIAA Member 
Subscripts

TCF

TS wall-normal direction

spanwise direction (parallel to the leading edge)

direction normal to $x$-direction

band-pass filtered in traveling crossflow frequency range (80 to $200 \mathrm{~Hz}$ )

band-pass filtered in TS frequency range $(200$ to $800 \mathrm{~Hz}$ )

\section{Introduction}

T AMINAR flow control remains a promising technique for improving the fuel efficiency of aircraft in the Unear future. In theory and in the laboratory, laminar flow control (LFC) techniques can work quite well. However, real-world applications result in additional difficulties that can be detrimental if not well-managed. For example, disruption of laminar flow can occur if the wing surface contains any small protuberances or surface imperfections. These imperfections can result from insect residue, rivets, bolts, steps, gaps, paint, and other sources. In order for LFC to effectively delay transition in an operational environment, we need to gain a better understanding of how surface irregularities affect transition. This is important so that we can specify more reasonable manufacturing tolerances.

Stationary crossflow typically dominates the transition process in swept-wing flows. However, steps can act as receptivity sites for other types of disturbances. Steps can also modify the mean flow in a way that destabilizes those disturbances for a short region downstream of the step.

Researchers have recently begun to study the effect of 2D steps on swept-wing transition in more detail. In earlier work, the results were limited to observations of the behavior of the transition front as the step height changed 102 More recently, researchers have begun to study the flow in more detail. Eppink et al! 3 reported detailed boundary-layer measurements downstream of a backward-facing step and identified several different types of instabilities that were introduced by the step and modulated by the stationary crossflow vortices. One of the unsteady disturbances identified downstream of the step is believed to be a traveling crossflow

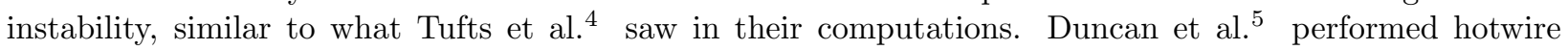
measurements downstream of forward- and backward-facing steps to determine the effect of the steps on stationary crossflow instabilities. They found that the steps caused an increase in N-factor for the stationary crossflow, but the stationary crossflow amplitudes were very low at the step, and therefore the uncertainty of the N-factors was high. Tufts et al. ${ }^{4}$ performed computations to study the interaction between stationary crossflow instabilities and a two-dimensional step excrescence. They found that the backward-facing step did not amplify the stationary crossflow modes, but they did verify the existence of a traveling instability in the recirculation region downstream of the step. They concluded that transition is likely caused by an interaction between this traveling mode and the stationary crossflow mode. Saeed et al 6 performed detailed hotwire measurements downstream of $2 \mathrm{D}$ strips placed on a swept-wing model. The strip is essentially a forward-facing step followed by a backward-facing step. They found that strips placed farther downstream generated larger amounts of unsteadiness, and concluded that the more developed stationary crossflow was interacting with traveling-crossflow disturbances, leading to earlier transition.

The complicated transition process involving protuberances (such as 2D steps) apparently involves the interaction of multiple types of disturbances. The current research is a follow-on experiment to the hotwire results described by Eppink et al $!^{3}$ and the stereo particle image velocimetry results described by Eppink and Yao 7 In the current work, we undertake time-resolved particle image velocimetry (TRPIV) measurements in order to gain a better understanding of the instability interactions and the breakdown mechanism leading to transition downstream of a backward-facing step.

\section{Previous Experimental Results}

Eppink et al $!^{3}$ and Eppink and $\mathrm{Yad}^{7}$ discuss the previous hotwire and PIV measurements for the backward-facing step (BFS) cases in detail, but we will summarize the results here as context for the following discussion. Hotwire measurements were performed with and without discrete roughness elements (DREs) on the leading edge of the model. In all cases, transition occurred far downstream and was dominated by stationary crossflow instabilities until the step height was increased above approximately $49 \%$ of the local boundary-layer thickness $(\delta)$ for the baseline case. At that step height, transition moved upstream abruptly, 
but still occurred more than 300 step heights downstream of the step. This was also well downstream of the separation bubble, which extended approximately 30 step heights downstream of the step. The hotwire data revealed a rich velocity spectrum downstream of the step, with unsteady disturbances in a broad frequency band $(f \approx 80$ to $1500 \mathrm{~Hz}$ ). These unsteady disturbances did not occur in the baseline case and are believed to be directly responsible for transition in the presence of the step since the stationary crossflow amplitudes remained too low to cause transition via their high-frequency secondary instabilities. Using simultaneous measurements from a second fixed hotwire, the unsteady disturbances were categorized as three distinct disturbance types: a traveling-crossflow (TCF) instability (80 to $200 \mathrm{~Hz}$ ), a Tollmien-Schlichting (TS) instability $(200$ to $800 \mathrm{~Hz}$ ), and a shear-layer instability ( 800 to $1500 \mathrm{~Hz}$ ). The traveling crossflow instability was found to have a wavelength of approximately $50 \mathrm{~mm}$, which is about 4 to 5 times larger than the predicted most unstable traveling crossflow mode for the no-step case. The TS instabilities were not present in the nostep case, and were likely amplified due to the short pressure gradient region caused by the backward-facing step.

The stationary crossflow modulated the unsteady disturbances in the spanwise direction, resulting in regions of peaks and valleys in fluctuation amplitude with spanwise spacing corresponding to the dominant stationary crossflow mode wavelength. Large spikes in velocity occurred well upstream of the breakdown location and appeared to be the manifestation of a type of breakdown mechanism resulting from the unsteady disturbances. The hotwire time trace in Fig. 1a shows an example of a location which experienced a large number of positive velocity spikes.

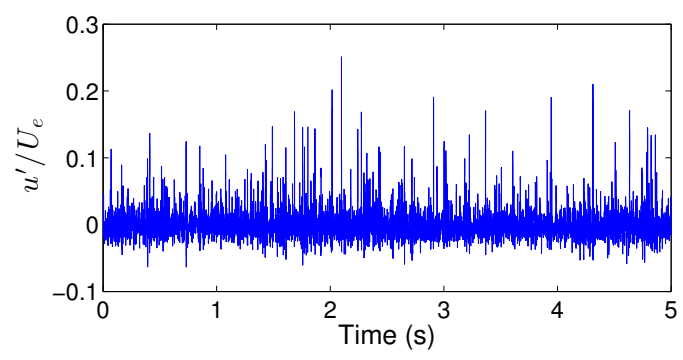

(a) Hotwire time trace in boundary layer showing positive velocity spikes.

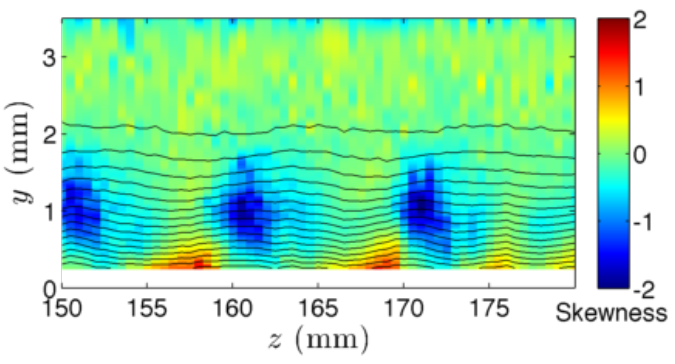

(b) Skewness (colors) and mean flow (lines) contours at a plane downstream of the step.

Figure 1: Results from hotwire campaign showing measurements of the spike breakdown mechanism.

Regions of positive and negative spikes occurred with spanwise spacing related to the stationary crossflow wavelength of $11 \mathrm{~mm}$. Positive spikes occurred near the wall while the negative spikes occurred off the wall and offset spanwise from the positive spikes. This is illustrated in Fig. 1b, in which positive skewness indicates the occurrence of positive spikes, and negative skewness indicates negative spikes. Stereo PIV measurements revealed that these spike events were not simply local events. In fact, these events occurred at the same time across multiple wavelengths of the stationary crossflow instability, in a way that increased the spanwise modulation initially caused by the stationary crossflow instability, as illustrated in Fig. 2

\section{Experimental Setup}

The experiment was performed in the 2-Foot by 3-Foot Low Speed Boundary-Layer Channel at NASA Langley Research Center. The tunnel is a closed circuit facility with a $0.61-\mathrm{m}$ high by $0.91-\mathrm{m}$ wide by $6.1-\mathrm{m}$ long test section. The tunnel can reach speeds up to $45 \mathrm{~m} / \mathrm{s}$ (unit Reynolds number, $R e^{\prime}=2.87 \times 10^{6} / \mathrm{m}$ ) in the test section. Freestream turbulence intensity levels, $T u=\frac{1}{U_{\infty}} \sqrt{\frac{1}{3}\left(u^{\prime 2}+v^{\prime 2}+w^{\prime 2}\right)}$, were measured using a crosswire in an empty test section to be less than $0.06 \%$ for the entire speed range of the tunnel, and less than $0.05 \%$ for the test speed of $26.5 \mathrm{~m} / \mathrm{s}$. This value represents the total energy across the spectrum, high-pass filtered at $0.25 \mathrm{~Hz}$. Thus, this tunnel can be considered a low-disturbance facility for purposes of conducting transition experiments $\frac{8}{8}$

The 0.0127-m thick flat plate model consists of a $0.41-\mathrm{m}$ long leading-edge piece, swept at $30^{\circ}$, and a 


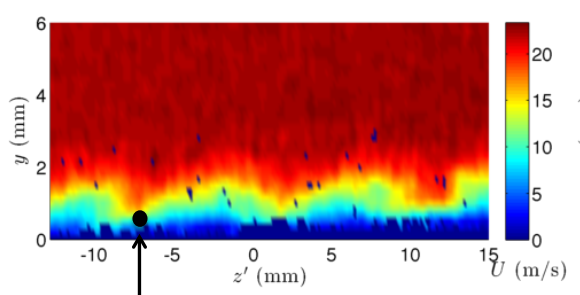

(a) Spike Event

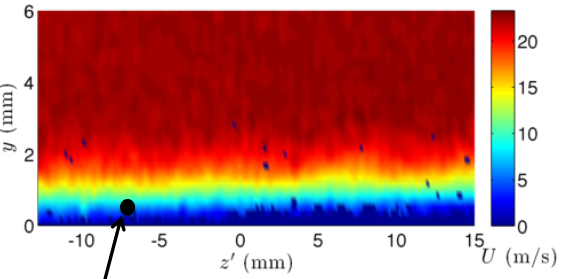

(b) Non-spike Event

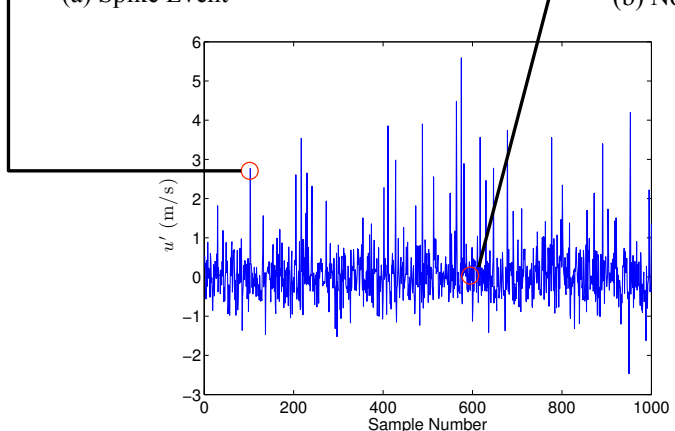

(c) PIV data from single point $\left(z^{\prime}=-7 \mathrm{~mm}, \mathrm{y}=0.7 \mathrm{~mm}\right)$

Figure 2: Instantaneous PIV snapshots at $x_{s h} \approx 120$ showing spike and non-spike events.

larger downstream piece (see Fig. 33). The model is $0.91 \mathrm{~m}$ wide (thus, spanning the width of the test section) and $2.54 \mathrm{~m}$ long on the longest edge. The downstream or leading edge pieces can be adjusted relative to each other using precision shims to create either forward-facing or backward-facing 2D steps of different heights, parallel with the leading edge. The leading edge piece was polished to a surface finish of $0.2 \mu \mathrm{m}$, and the larger downstream plate had a surface finish of $0.4 \mu \mathrm{m}$. A leading-edge contour was designed for the bottom side of the plate in order to make the suction-peak less severe, and therefore avoid separation, which could potentially cause unsteadiness in the attachment line.

A 3D pressure body along the ceiling was designed to induce a streamwise pressure gradient, which, along with the sweep, causes stationary crossflow growth. A second purpose of the ceiling liner was to simulate infinite swept-wing flow within a mid-span measurement region of width 0.3 meters. This was achieved by designing the liner such that the $C_{p}$ contours were parallel with the leading edge within the measurement region. The ceiling liner was fabricated out of a hard foam using a computer-controlled milling machine.

All measurements were performed at a freestream velocity of $26.5 \mathrm{~m} / \mathrm{s}\left(R e^{\prime}=1.69 \times 10^{6} / \mathrm{m}\right)$, and with a backward-facing step height of $1.184 \mathrm{~mm}(49 \% \delta)$. The current experiment utilized a single leading-edge roughness configuration consisting of DREs with a diameter of $4.4 \mathrm{~mm}$. The DREs were applied with a spanwise spacing, $\lambda_{z}$, of $11 \mathrm{~mm}$ and were approximately $20 \mu \mathrm{m}$ thick. The spacing of the DREs $(11 \mathrm{~mm})$ corresponds to the most amplified stationary crossflow wavelength calculated for the baseline case with no step. For more details of the experiment setup, refer to Eppink .9

A high-speed double-pulsed Nd:YAG laser provided the laser sheet for the time-resolved particle-image velocimetry (TRPIV) measurements (see Fig. 3). The laser sheet was set up parallel with the leading edge and the backward-facing step. Two high-speed 4 megapixel cameras were used to acquire the images. One was placed on the outboard side of the test section at approximately $30^{\circ}$ to the laser sheet, and the second camera was placed on the inboard side (in backward scattering) at an angle of approximately $45^{\circ}$ to the laser sheet (Fig. 3). To achieve the desired field of view and resolution, $300 \mathrm{~mm}$ lenses were utilized, resulting in a total possible measurement area of approximately $60 \mathrm{~mm} \mathrm{x} 30 \mathrm{~mm}$. The area of interest was reduced to approximately $37 \mathrm{~mm} \times 5 \mathrm{~mm}$ to obtain an acquisition rate of $4.7 \mathrm{kHz}$. This area allowed acquisition of approximately three wavelengths of the stationary crossflow instability in a single frame, while still acquiring approximately 30 points (using $75 \%$ overlap and $24 \times 24$ pixel interrogation size) inside the boundary layer. The cameras and laser were all mounted on the same traversing system, which allowed measurements at multiple locations with relative ease. An oil-based fog machine generated the seeding, which was introduced downstream of the test section. 


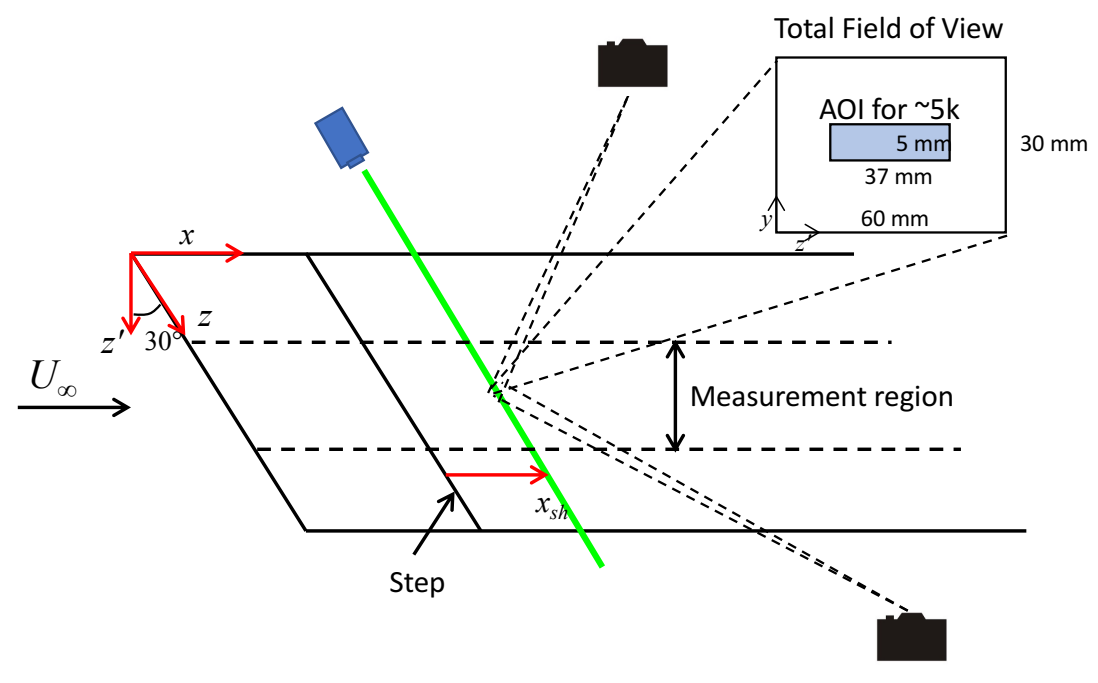

Figure 3: Top view of PIV setup.

\section{Data Analysis}

Data acquired at each streamwise station consisted of 10,000 images at a frame rate of $4.7 \mathrm{kHz}$. Since this is a stereo configuration, all three velocity components were acquired. The velocity components acquired from the initial processing of the images were in the direction normal to the step $\left(x_{c}\right)$, normal to the wall $(y)$, and parallel to the step $(z)$. The velocity components in each frame were first interpolated to remove any dropout points. Then the $x_{c}$ and $z$ components were transformed into the components parallel and normal to the free stream direction $\left(x\right.$ and $\left.z^{\prime}\right)$, for further analysis.

For analysis of the two different unsteady instabilities (traveling crossflow and TS), the time series data at each point were filtered around the two frequency bands ( 80 to $200 \mathrm{~Hz}$ and 200 to $800 \mathrm{~Hz}$ ) by performing zero-phase filtering using a 4th-order Butterworth band-pass filter. Due to the noisiness of the signal, the bandpass-filtered data were also smoothed in space using a 2-D Gaussian smoothing kernel with a standard deviation of 4 in the $z$-direction, and 2 in the $y$-direction. This smoothing was applied to aid in the detection and visualization of the 2D flow structures that were visible from the TRPIV data. A rectangular kernel was used since the flow structures are typically larger in the spanwise direction compared to the wall-normal direction.

Proper orthogonal decomposition (POD) was also utilized throughout the paper to help identify flow structures. This method, first proposed by Lumley, 10 is commonly used in the analysis of PIV results to identify the large-scale flow structures that are present in the flow. The input flow field data are deconstructed into a set of POD modes, which can be used to partially or fully reconstruct the flow field. The modes are orthogonal and are optimized such that the smallest number of modes captures the largest fraction of the total energy of the flow field. The modes are ranked based on their energy content, so that the first mode contains the most energy throughout the entire data set, and so on.

POD is used in the current work for two main purposes. The first is to reconstruct the flow field in time from a small number of POD modes so that the development of the traveling crossflow and TS instabilities can be studied and more easily visualized (Section VI). Without using POD reconstruction, the results are noisy and it is difficult to obtain useful isosurfaces, particularly when the amplitudes of the instabilities are low near the step. The POD reconstruction for a specific snapshot is performed by summing up the first 5 modes multiplied by their respective coefficients for that snapshot. The second use of POD in this paper is to identify patterns that occur in the traveling crossflow and TS instabilities during specific flow field events (Section VII). 


\section{Comparison of TRPIV and Hotwire Data}

A comparison between the mean PIV results from the low-speed stereo PIV system and the hotwire results was presented by Eppink and Yao. ${ }^{7}$ Overall, the PIV and hotwire results agreed well. In this section, we will focus on a comparison of the fluctuating velocity component. Velocity spectra acquired at several different streamwise locations downstream of the step at $y=1 \mathrm{~mm}$ are shown in Fig. 4 . These spectra were averaged across the span in order to get a sense of the overall unsteady flowfield. Results from the hotwire data are shown in Fig. 4a, while results from similar streamwise locations are shown in Fig. $4 \mathrm{~b}$ for the TRPIV measurements. Figure $4 \mathrm{~b}$ also includes a single hotwire spectra (from $x_{s h}=69$ ) so that a direct comparison can be made. Note that the y-scales are different between the two plots. The TRPIV data have a much higher noise floor than the hotwire data (about 5 to 6 orders of magnitude), which is to be expected. However, we can still clearly discern two broad peaks between 80 to $200 \mathrm{~Hz}$ and 200 to $800 \mathrm{~Hz}$ in the TRPIV spectra corresponding to the traveling crossflow and TS frequency bands. These two frequency ranges are denoted by the vertical black lines in both figures. In general, the TRPIV spectra are larger in amplitude compared to the hotwire spectra from similar locations. This is especially true for the locations closer to the step, where the amplitudes are lower, such as $x_{s h} \approx 20$. Notice also that there is not very much energy present in the higher frequency band $(800$ to $1500 \mathrm{~Hz})$ in the TRPIV spectra, which was associated with the shear-layer instability from the hotwire data. Despite the discrepancy in amplitudes and the lack of resolution of the higher-frequency modes, it is quite encouraging that we are even able to measure these fluctuations at all using TRPIV, since they are of fairly low amplitude.

One question that arises when performing TRPIV is the ability of the particles to respond to the high frequency perturbations in the flow. Using the approach of Mei $\frac{11}{11}$ we can estimate a low-pass frequency cut-off of the particles, assuming a certain particle diameter and density ratio. If we assume the particle density is approximately that of water, and the particle diameter ranges from 0.5 to $2 \mu \mathrm{m}$, the estimated low-pass frequency cutoff ranges from 14 to $227 \mathrm{kHz}$. Thus, the particles in this flow should be able to respond to frequencies in the 800 to $1500 \mathrm{~Hz}$ range.

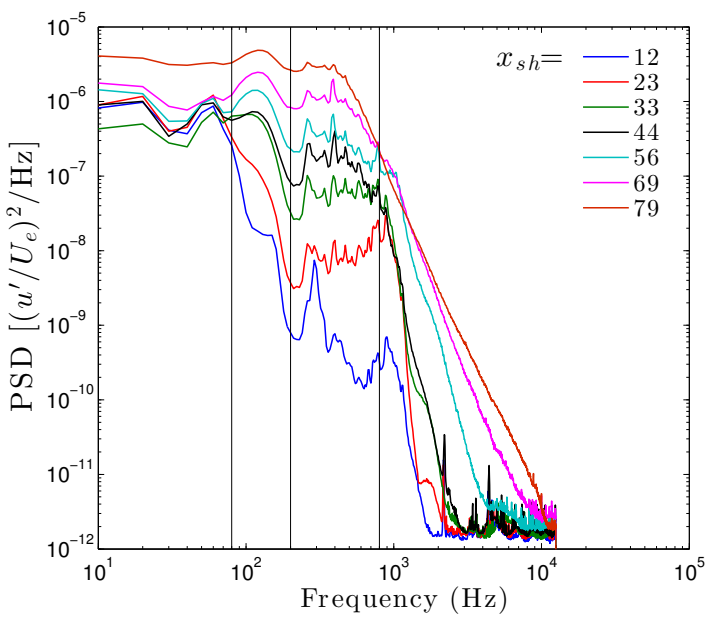

(a) Hotwire

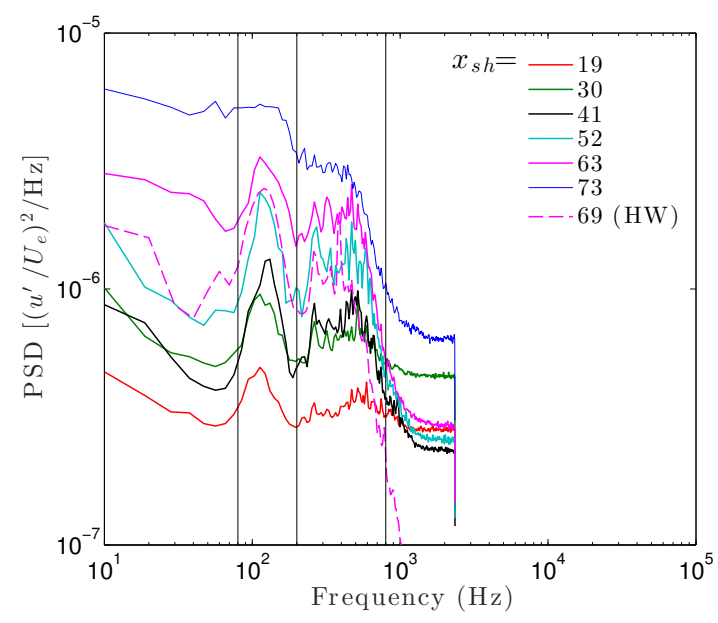

(b) TRPIV

Figure 4: Comparison of spanwise-averaged velocity spectra from hotwire and TRPIV results at several streamwise locations downstream of the step.

The TRPIV and hotwire results are further compared in Fig. 5. in which color contours of $u_{r m s}^{\prime}$ amplitudes are plotted within the traveling crossflow and TS frequency bands across the full $y-z$ plane. Unfortunately, we do not have full planes of hotwire data and TRPIV data for the same DRE case at the same streamwise location, but we can still qualitatively compare results from different streamwise locations. Fig. 5 shows TRPIV data at $x_{s h}=73$ and hotwire data at $x_{s h}=100$. The amplitudes for the TRPIV data are higher than that of the hotwire data, even though the TRPIV data were acquired upstream of the hotwire data, where amplitudes should be lower. This agrees with the general trend from the spectra. However, we still observe 
the same modulation patterns due to the interaction of the stationary crossflow with the different unsteady instabilities. The $u_{r m s}^{\prime}$ contours for the traveling crossflow frequency band exhibit a modulation pattern with a wavelength of half of the primary stationary crossflow mode wavelength of $11 \mathrm{~mm}$, with some peaks in amplitude occurring near the wall, and the adjacent peaks occurring slightly off of the wall. This pattern is similar between the TRPIV and hotwire data (Figs. 5a and 5c). The $u_{r m s}^{\prime}$ contours for the TS frequency band in both cases (Figs. $5 \mathrm{~b}$ and $5 \mathrm{~d}$ ) are strongly modulated with a wavelength of $11 \mathrm{~mm}$, equal to that of the primary stationary crossflow mode. Overall, the qualitative agreement between the TRPIV and hotwire data is encouraging. Despite the discrepancy in amplitude of the fluctuating component, the results can still provide valuable insight into the instability interactions that occur downstream of the step.

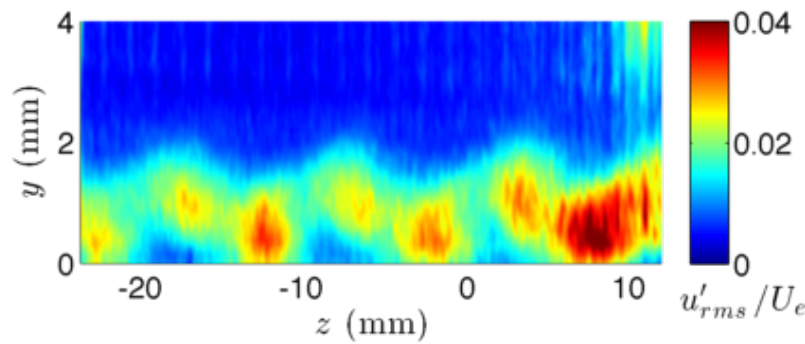

(a) TRPIV, TCF, $x_{s h}=73$

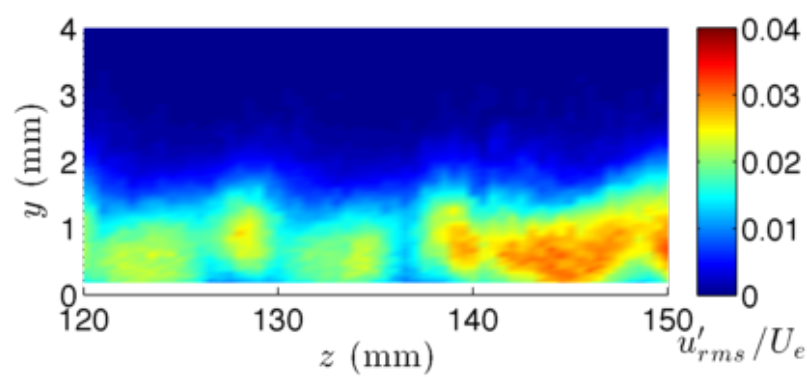

(c) Hotwire, TCF, $x_{s h}=100$

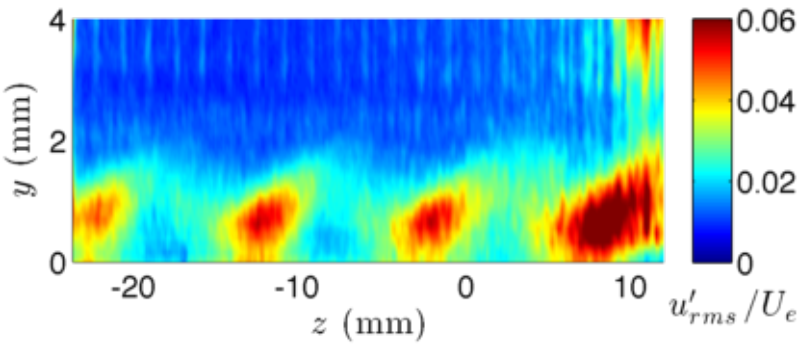

(b) TRPIV, TS, $x_{s h}=73$

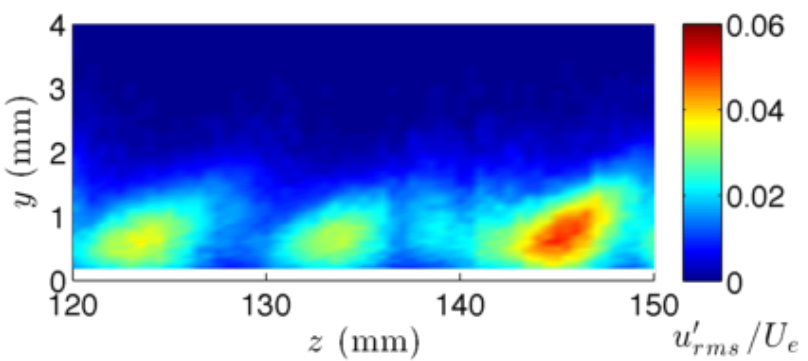

(d) Hotwire, TS, $x_{s h}=100$

Figure 5: Comparison of fluctuating velocity RMS for traveling crossflow and TS frequency bands.

\section{Development of Unsteady Instabilities}

In this section, the downstream development of the traveling crossflow and TS disturbances is examined. As mentioned in the previous section, the higher-frequency band associated with the shear-layer instability was not well-resolved with the TRPIV system due to the higher noise level. Additionally, this instability is of lower amplitude than the TCF and TS instabilities, and therefore is not believed to be as important in the transition process downstream of the step. Due to these reasons, analysis of this higher-frequency instability is not included in this paper, and the discussion instead focuses on the two lower frequency instabilities.

Data were acquired at several stations downstream of the step, starting as close to the step as physically possible, given the finite thickness of the laser sheet and the inevitable reflections that occur near the step. This location is estimated to be approximately $1 \mathrm{~mm}$ downstream of the step, though it is not possible to obtain an exact location since we do not know precisely where the center of the laser sheet is located. The fluctuation amplitudes at this station were so low compared to the noise level that there was nothing of interest to report. Therefore, the measurements reported in this paper begin at $x_{s h}=9$ and go downstream to $x_{s h}=73$ in approximately $13 \mathrm{~mm}$ increments.

POD was performed individually on the traveling crossflow and TS band-pass-filtered data to examine the dominant flow structures of each instability. The POD energy spectra for the first 50 POD modes at all locations are shown in Fig. 6 for the traveling crossflow and TS data. The eigenvalues of the POD modes correspond to the kinetic energy content of the respective POD mode. Thus, the cumulative modal energy corresponds to the cumulative sum of the eigenvalues, normalized by the sum of all the eigenvalues. The 
plots indicate that at most stations (excluding the most upstream station), the first mode makes up at least $40 \%$ of the total modal energy. Moreover, the first 5 POD modes account for more than $90 \%$ of the total modal energy. The lower values of cumulative energy for the most upstream station $\left(x_{s h}=9\right)$ are believed to be due to the extremely low signal-to-noise ratio at this station, since the amplitudes of the disturbances were barely discernible.

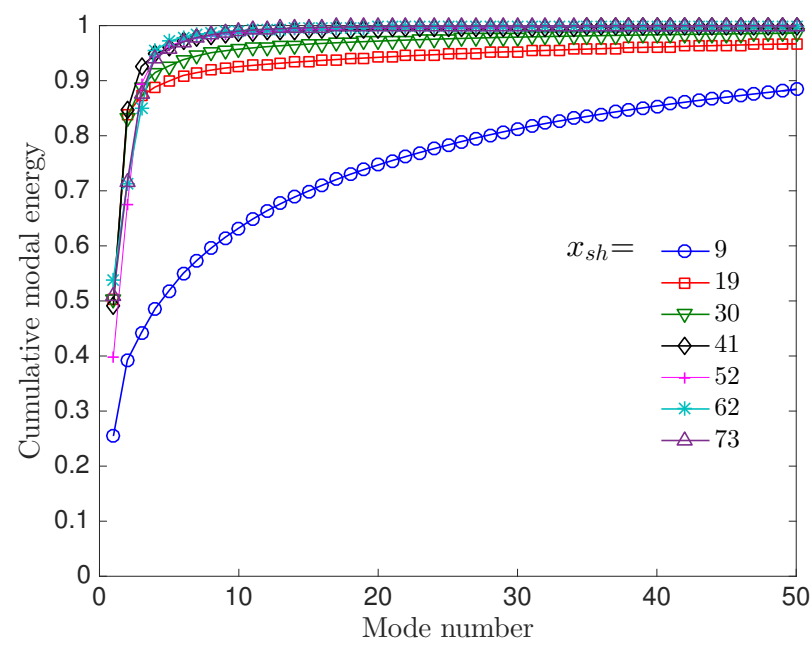

(a) TCF

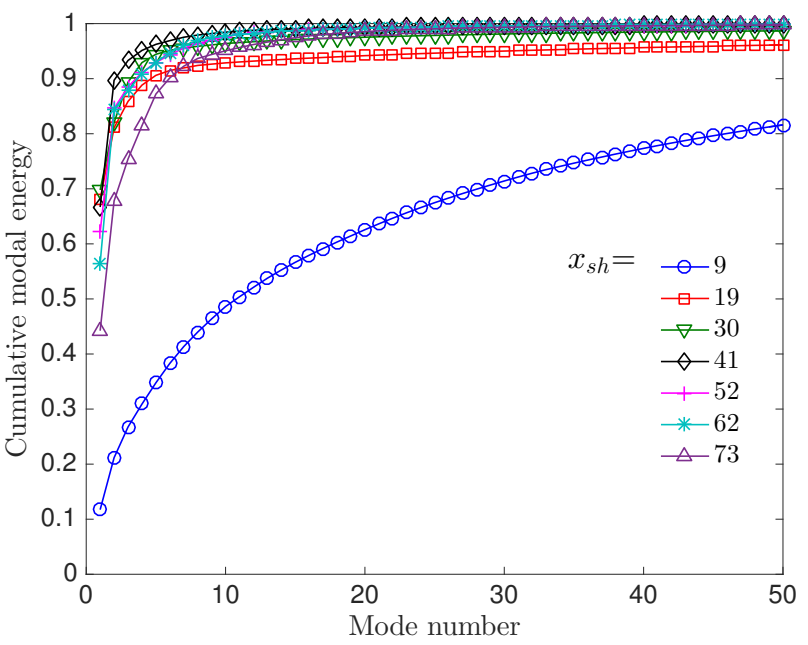

(b) TS

\section{Figure 6: Cumulative POD eigenvalues (energy) for the first 50 POD modes at each streamwise} location.

The first POD modes at each streamwise station for the traveling crossflow band-pass-filtered data are shown in Fig. 7 for all three components of velocity. The first POD modes for the three most upstream stations consist of a structure with a large spanwise wavelength of approximately $40 \mathrm{~mm}$. In the hotwire data presented by Eppink,, 9 the spanwise wavelength of the traveling crossflow was found to be approximately $50 \mathrm{~mm}$. The TRPIV measurement is less than the $50 \mathrm{~mm}$ wavelength found from the hotwire data, but still in good qualitative agreement with the findings. The stations downstream of reattachment, starting at $x_{s h}=41$, begin to exhibit some modulation of that primary large wavelength structure. Eventually, at the last two stations $\left(x_{s h}=62\right.$ and 73$)$, the first POD mode exhibits a strong $11 \mathrm{~mm}$ wavelength, and the large wavelength structure is no longer present.

The first POD modes at each streamwise station for the TS band-pass filtered data are shown in Fig. 8 The first mode at the most upstream station $\left(x_{s h}=9\right)$ for the TS band-pass-filtered data is mostly dominated by noise near the left edge of the figure. However, all of the stations downstream show a clear modulation of the TS instability by the primary stationary crossflow mode. The first POD mode for this instability does not change drastically downstream, but the modulation becomes stronger and the peaks more localized. 


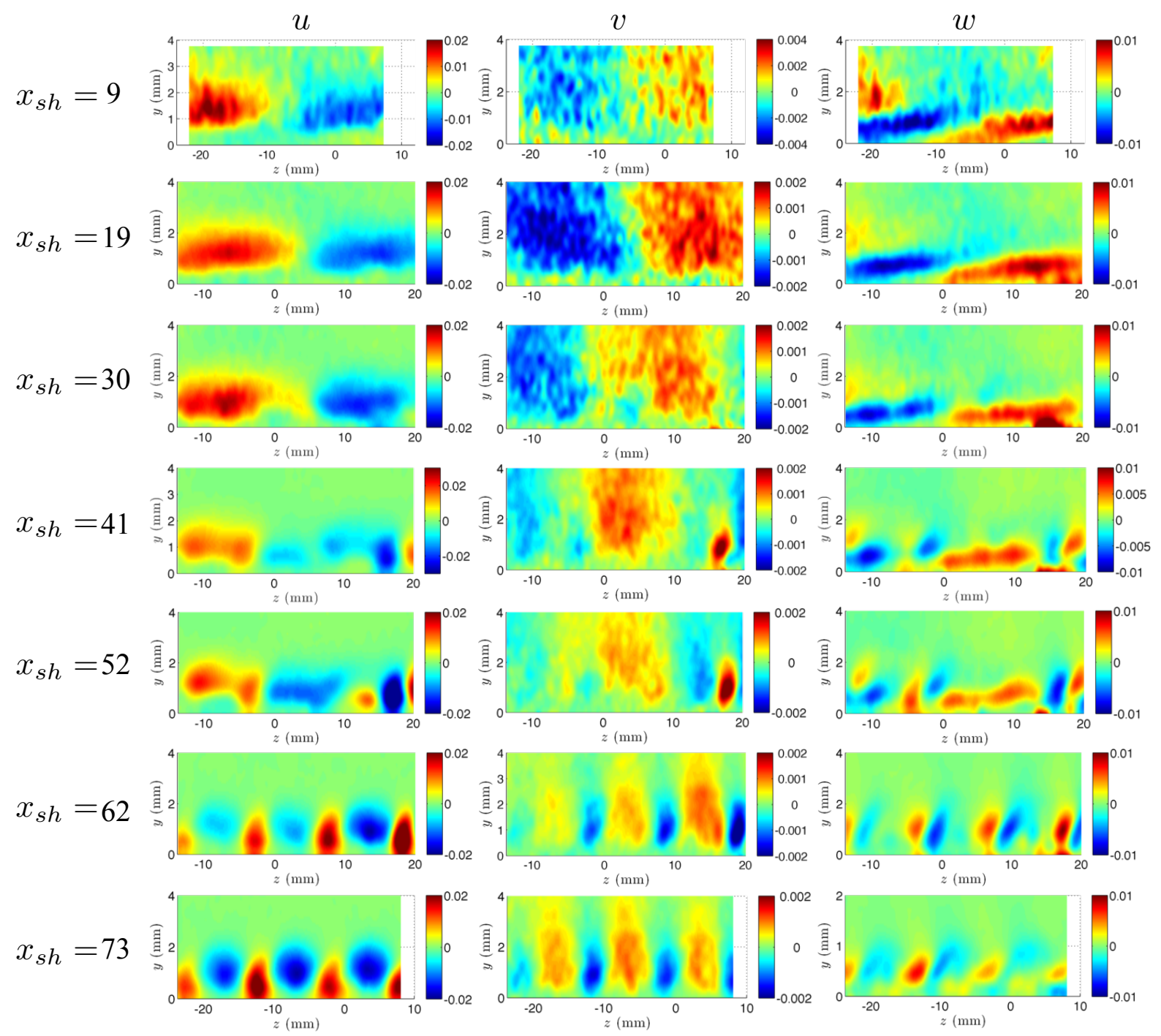

Figure 7: First POD modes at each streamwise station for traveling crossflow frequency band. 


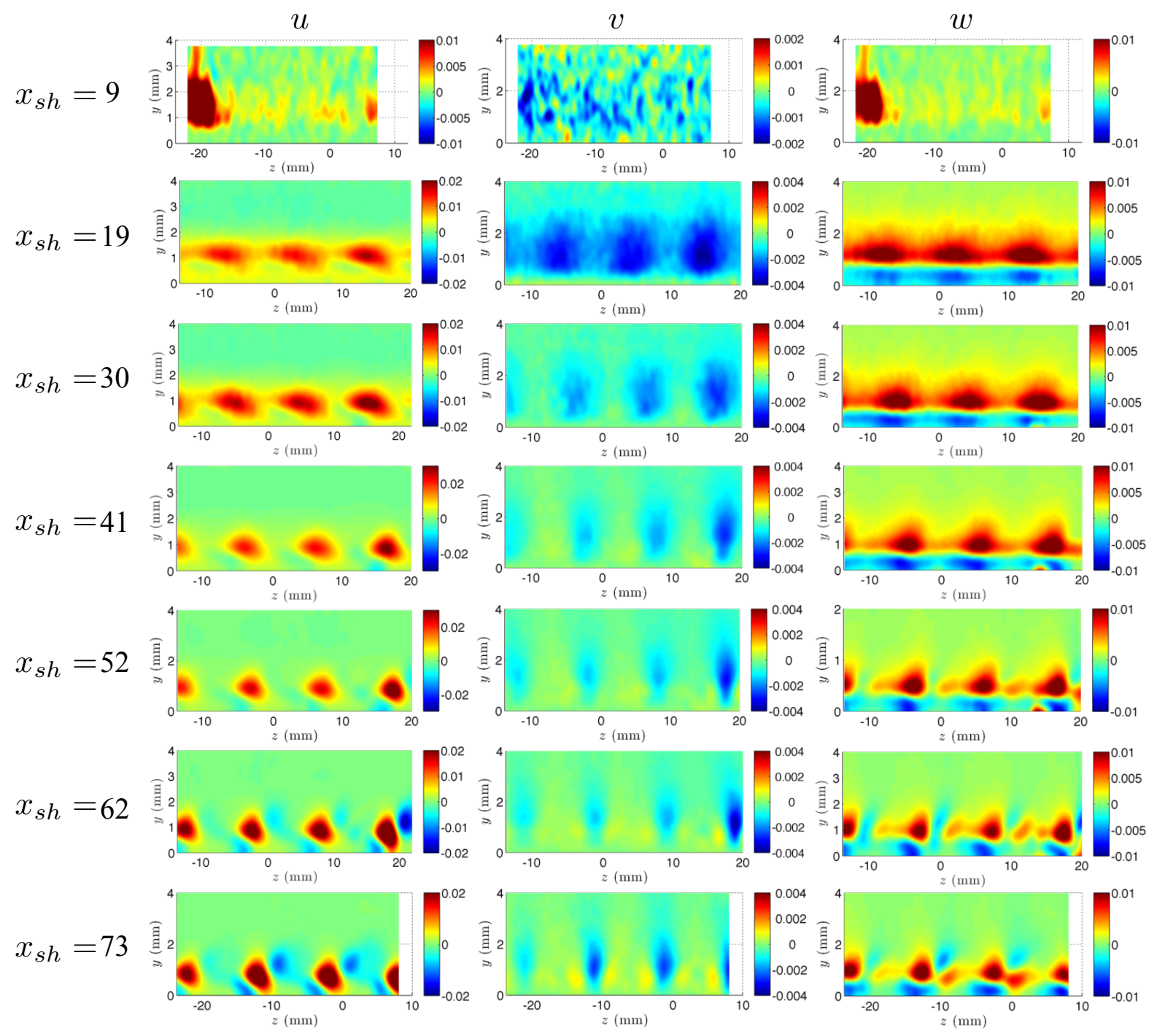

Figure 8: First POD modes at each streamwise station for TS frequency band.

Figure. 9 shows band-pass-filtered results at three sequential times for the traveling-crossflow frequency band at $x_{s h}=19$. The right column of this figure includes the reconstructed data at the same times using the first five POD modes. While the large traveling crossflow structure is visible in the bandpass-filtered data (left column), it is very noisy. The POD reconstruction (right column) provides a better visualization of the behavior of this instability. This instability travels from left to right primarily in the spanwise direction (in-plane), which is in agreement with the hotwire measurements.

Figure 10 shows the same results but for the TS frequency band. Once again, the TS structures are somewhat visible in the band-pass-filtered data, but the patterns become much more clear with the POD reconstruction. This mode, in contrast to the traveling crossflow mode, is traveling primarily out of plane. Therefore, there is not much in-plane motion visible, particularly at the most upstream stations, before it becomes strongly modulated by the stationary crossflow vortices. 


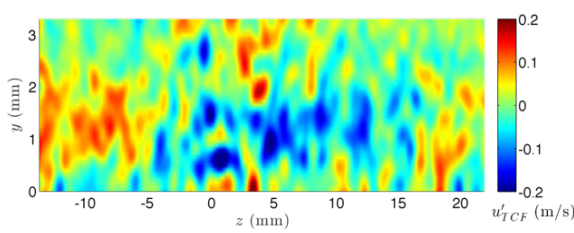

(a) $t=1.1787$ seconds

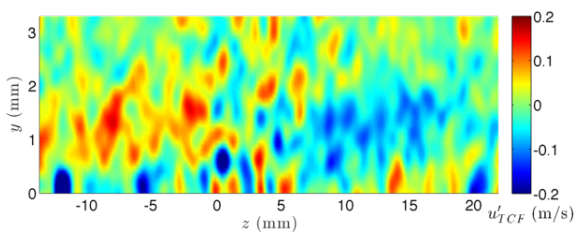

(c) $t=1.798$ seconds

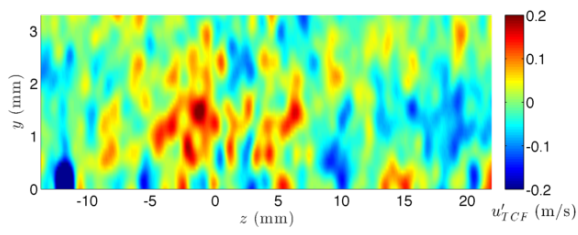

(e) $t=1.1809$ seconds

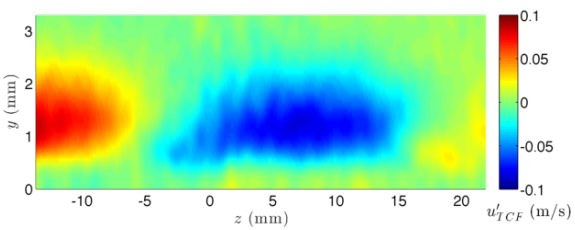

(b) $t=1.1787$ seconds

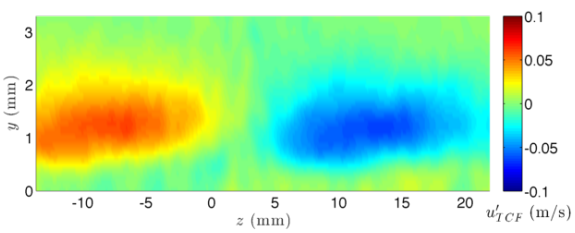

(d) $t=1.798$ seconds

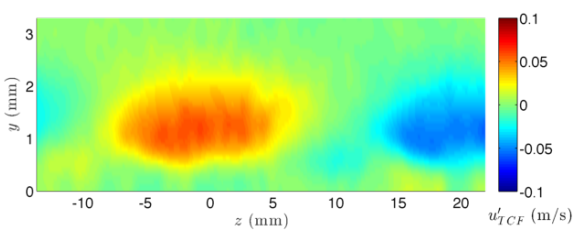

(f) $t=1.1809$ seconds

Figure 9: Band-pass filtered streamwise velocity in the traveling crossflow frequency range $(80$ to $200 \mathrm{~Hz})$ at $\mathrm{x}_{\mathrm{sh}}=19$ for three consecutive times. Figures in the left column are the bandpass-filtered data, the figures in the right column are the POD reconstruction of the bandpass-filtered data using the first 5 modes.

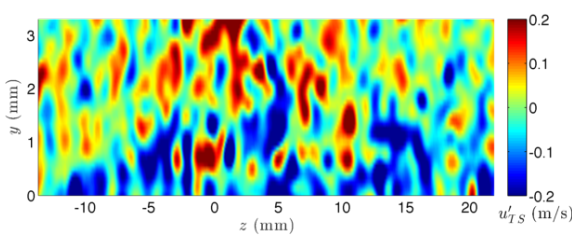

(a) $t=1.1787$ seconds

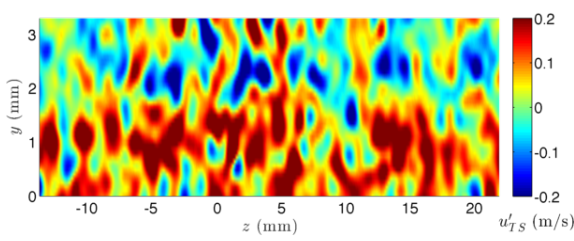

(c) $t=1.798$ seconds

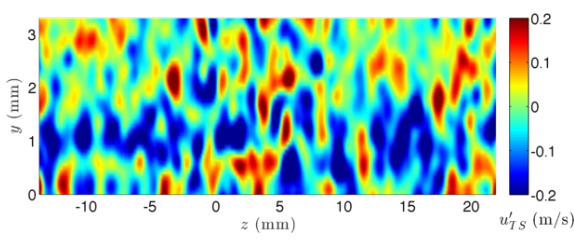

(e) $t=1.1809$ seconds

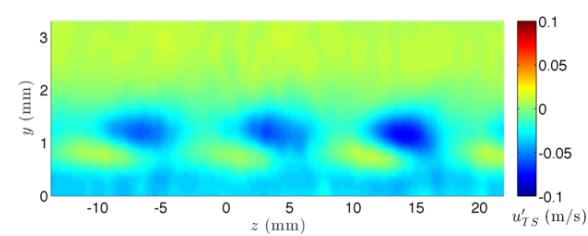

(b) $t=1.1787$ seconds

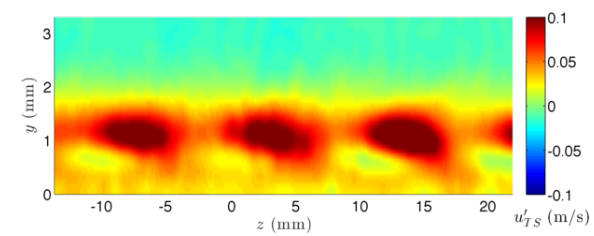

(d) $t=1.798$ seconds

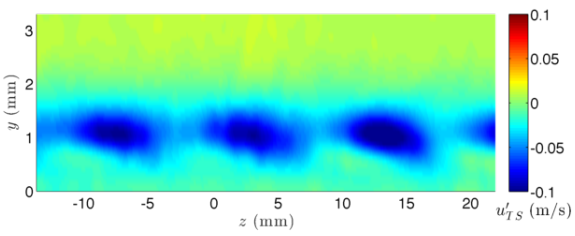

(f) $t=1.1809$ seconds

Figure 10: Band-pass filtered streamwise velocity in the Tollmien-Schlichting frequency range $(200$ to $800 \mathrm{~Hz})$ at $\mathrm{x}_{\mathrm{sh}}=19$ for three consecutive times. Figures in the left column are the bandpass-filtered data, the figures in the right column are the POD reconstruction of the bandpass-filtered data using the first 5 modes. 
We can further visualize the behavior of these two instabilities by expanding the results in three dimensions, using time as the x-axis, and plotting isosurfaces of the band-pass-filtered streamwise velocity. POD reconstruction using the first 5 modes is performed for 100 samples (constituting a total of $0.02 \mathrm{~s}$ ). The POD-reconstructed data are used here because the band-pass-filtered data are too noisy to obtain useful isosurfaces, particularly for the most upstream stations. The results are shown in Fig. 11 for the first three stations, and Fig. 12 for the last four stations. Note that these plots can be somewhat misleading, particularly for the traveling crossflow instability, since this instability is traveling primarily in plane. Thus, these are not the actual three-dimensional spatial structures that exist, this is simply a convenient way of visualizing the action of this instability over time in the $2 \mathrm{D}$ plane.

It should be noted that we cannot draw firm conclusions about the physical nature of the disturbances solely from these data since the data were only acquired in a single plane. Thus, it is not clear which direction the instabilities are traveling. However, we know from the hotwire results that, at least at the most upstream stations before the instabilities become highly modulated by the stationary crossflow, the traveling crossflow mode is traveling primarily in the spanwise plane, while the TS mode is traveling close to the chordwise direction.
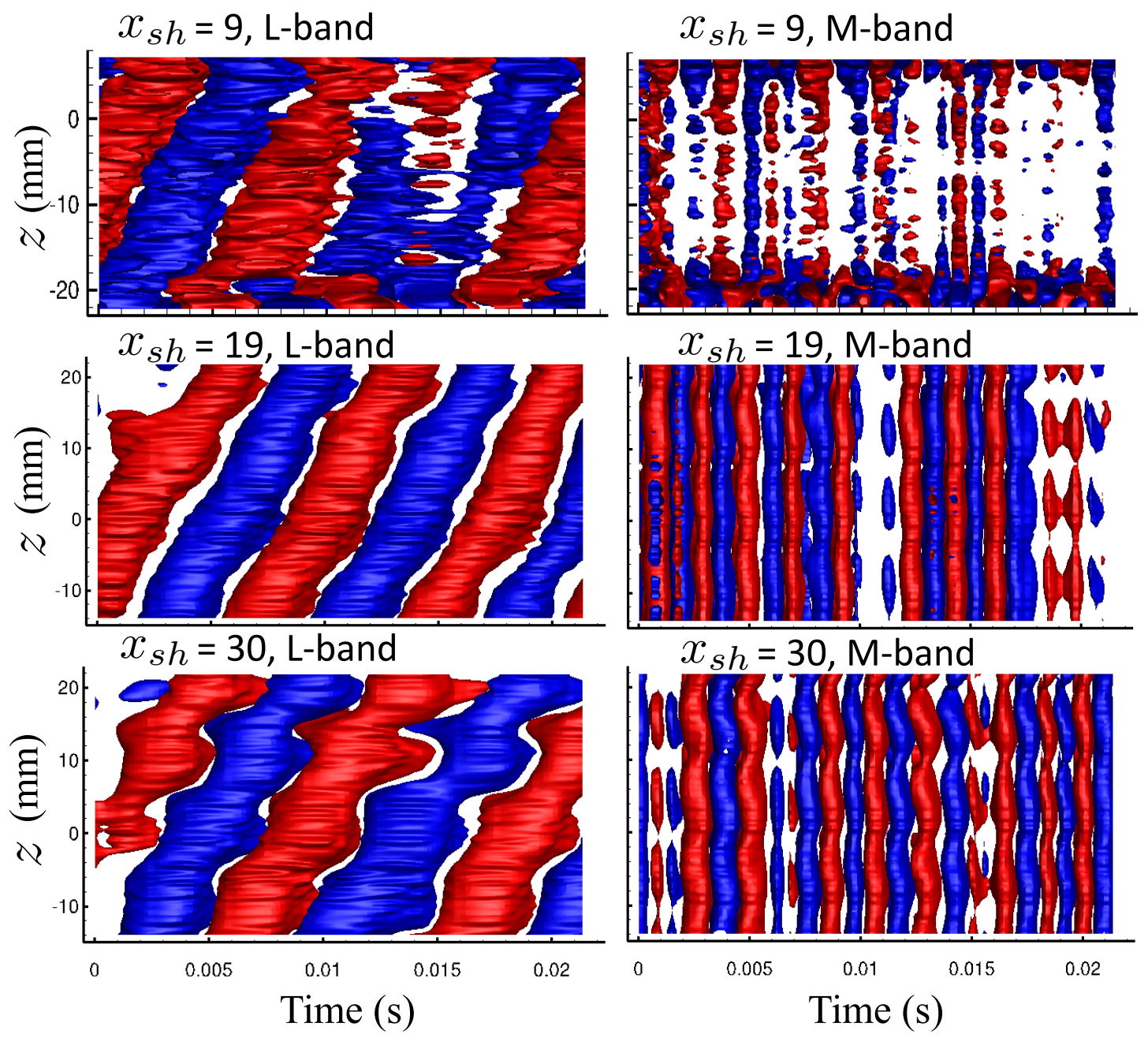

Figure 11: Traveling crossflow and TS streamwise velocity isosurfaces reconstructed using the first 5 POD modes and expanded in time. First three stations shown from $x_{s h}=9$ to 30 .

The traveling crossflow mode (left column) appears as a spanwise traveling disturbance as observed from Fig. 9 at the first two stations. Starting at $x_{s h}=30$ (Fig. 11), some modulation of the structures starts 
to become visible at the $11 \mathrm{~mm}$ primary stationary crossflow mode wavelength. This modulation becomes stronger and stronger downstream, until $x_{s h}=62$ (Fig. 12), where the large spanwise traveling structure from the upstream stations is no longer readily visible. The TS mode (right column) appears as a 2D, streamwisetraveling mode for the first three to four stations. Starting at $x_{s h}=19$, some minor spanwise modulation of the structure starts to become visible. This modulation becomes stronger downstream. By $x_{s h}=52$, the mode has become highly three-dimensional. In fact, it looks like there may be two oblique modes present, though it is not possible to tell from these results alone. Wasserman and Kloker ${ }^{[12}$ studied the interaction of TS and stationary crossflow instabilities in a flow with a pressure-gradient changeover. They found that the interaction resulted in the growth of a pair of oblique modes, which they referred to as low-frequency secondary modes. They also found that these modes experienced localized amplification in the spanwise plane due to the deformed primary state. We may be seeing a similar phenomenon in this flow.
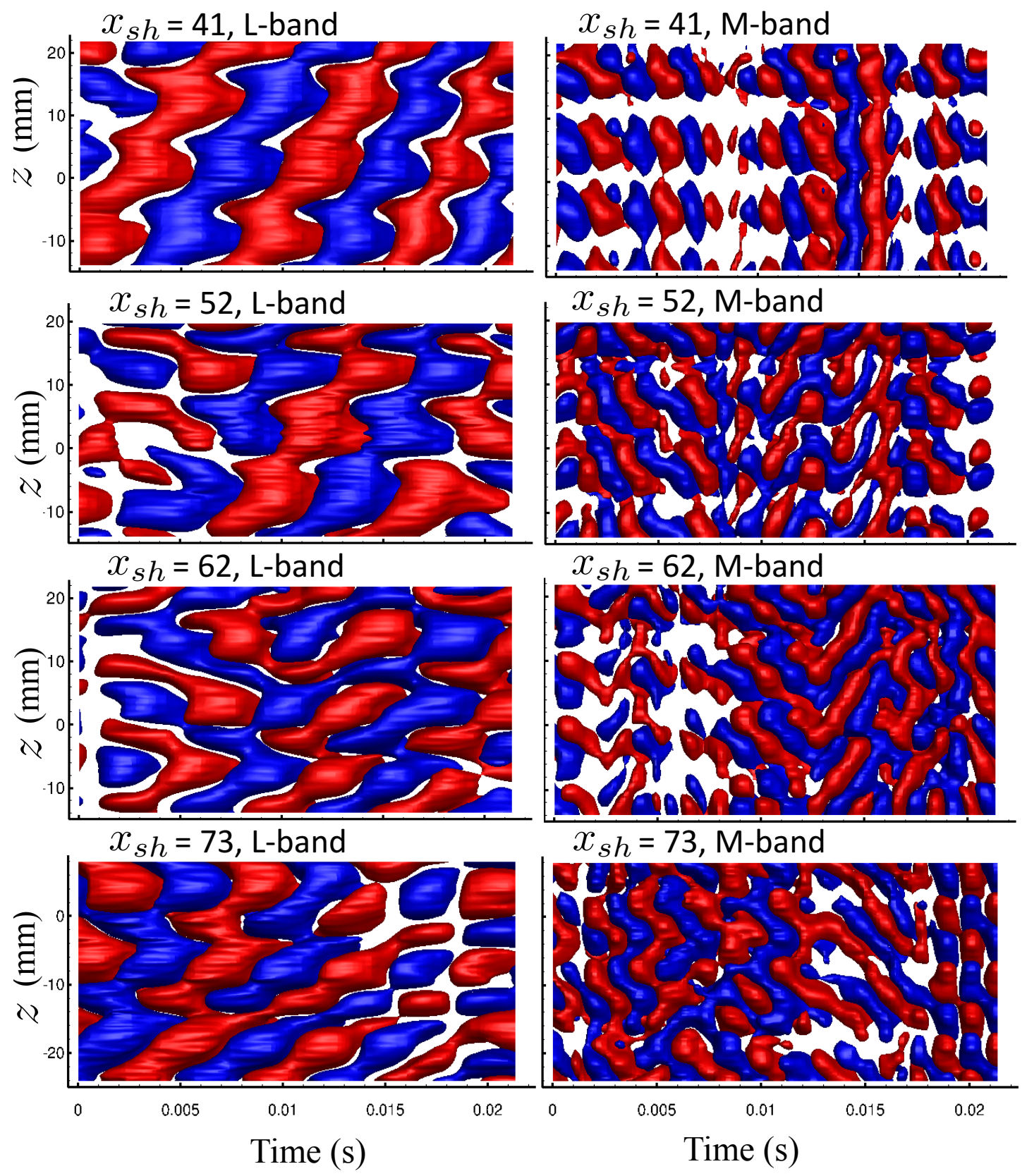

Figure 12: Traveling crossflow and TS streamwise velocity isosurfaces reconstructed using the first 5 POD modes and expanded in time. Last four stations shown from $x_{s h}=41$ to 73 . 


\section{Velocity Spikes}

The aforementioned large amplitude velocity spikes first become apparent at the $x_{s h}=52$ station. The data at this station are therefore analyzed further to attempt to gain a better understanding of this mechanism. The time-averaged streamwise velocity field is shown in Fig. 13 along with the skewness of the time-resolved data. The skewness, similar to the hotwire data (Fig. 1b), illustrates that positive spikes (indicated by positive skewness) occur mainly near the wall, while negative spikes occur off the wall and slightly offset in span from the locations of positive spikes. The time series from a single point $(z=-3 \mathrm{~mm}, y=0.5 \mathrm{~mm}$ in Fig. 13) is shown in Fig. 14 . This point is located in a region where positive velocity spikes occur.

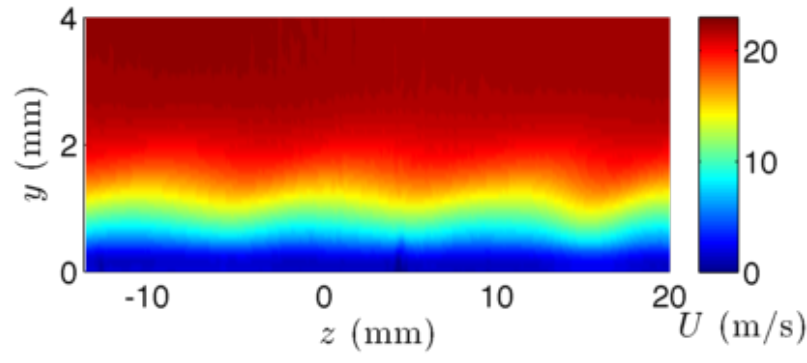

(a) Time-averaged streamwise velocity at $x_{s h}=52$.

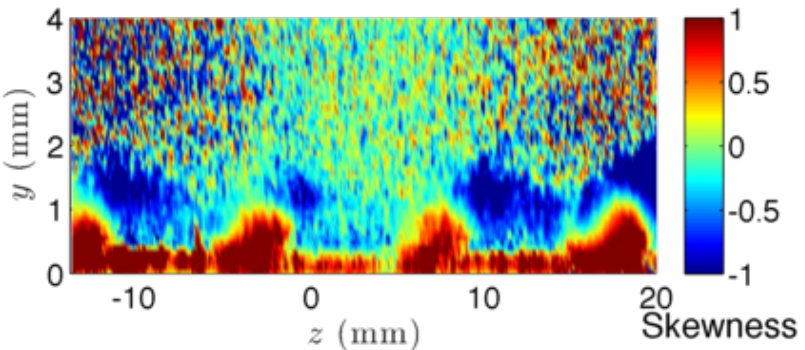

(b) Skewness of streamwise velocity at $x_{s h}=52$.

Figure 13: Mean flow and skewness at $x_{s h}=52$.

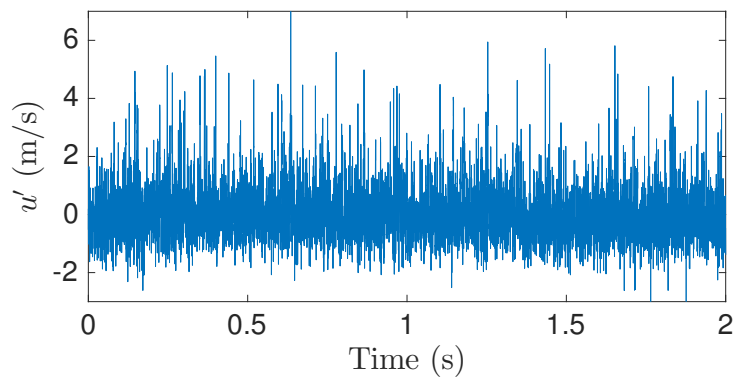

Figure 14: Time trace at $x_{s h}=52, y=0.5 \mathrm{~mm}, z=-3 \mathrm{~mm}$.

A single snapshot during one of the large velocity spikes is shown in Fig. 15, which includes the streamwise velocity along with the band-pass-filtered streamwise velocity for both the traveling crossflow and TS frequency bands. At this streamwise location, the stationary crossflow has caused a strong modulation of the traveling crossflow, which is evident in Fig. 12. During the spike event, the traveling crossflow has reached a particular point in the phase such that it is positive near the wall and negative away from the wall. These positive and negative regions occur in certain spanwise locations such that the overall effect is an enhancement of the modulation caused by the stationary crossflow. The stationary crossflow also modulates the TS instability, but in a different manner. At this streamwise location, the initially 2D TS wavefront has become distorted such that the negative and positive parts of the phase occur at the same time in this spanwise plane. The motion (which is illustrated by Eppink et al ${ }^{13}$ using phase-locked hotwire results), is such that the positive peak occurs off the wall, then moves toward the wall and to the left. Then the negative peak does the same.

Note that there are regions where the positive parts of the traveling crossflow and TS instabilities occur simultaneously (such as near the wall at $z \approx-3$ ). The same is true of the negative parts (such as $z \approx 0$, $y \approx 1$ ). These correspond to the regions where the positive and negative spikes occur, respectively. In other words, when the phases of the traveling crossflow and TS instabilities align in time and space, the large spikes in velocity occur. However, when the traveling crossflow reaches the opposite part of its cycle (i.e., $180^{\circ}$ out of phase with Fig. 15b, such that it counteracts the spanwise modulation caused by the stationary crossflow, the spikes do not occur, even when the TS and traveling crossflow instabilities align in space. This is why we only observe positive and negative spikes in certain spatial areas. To further confirm these 
observations, we perform POD analysis. First, we will look at the POD modes for the entire set of 10,000 snapshots. Second, we will perform the POD analysis on a subset of these snapshots that correspond to the times when the large velocity spikes occur.

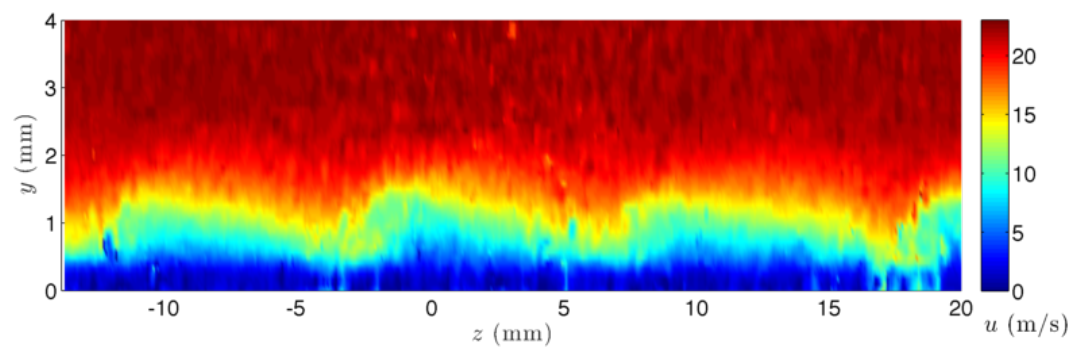

(a) Unfiltered

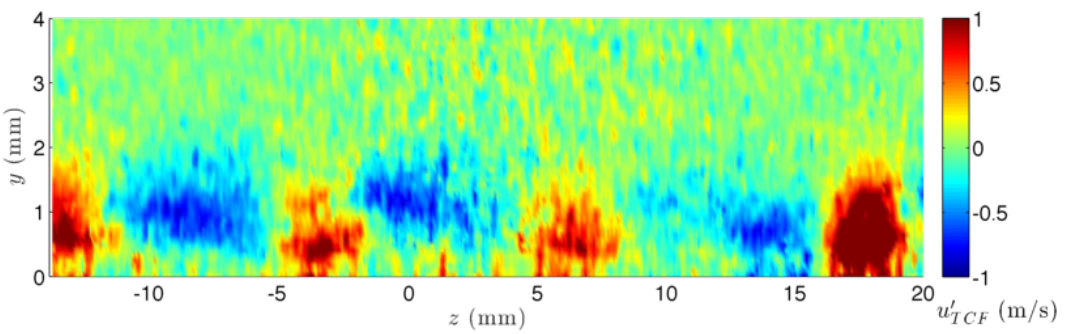

(b) TCF

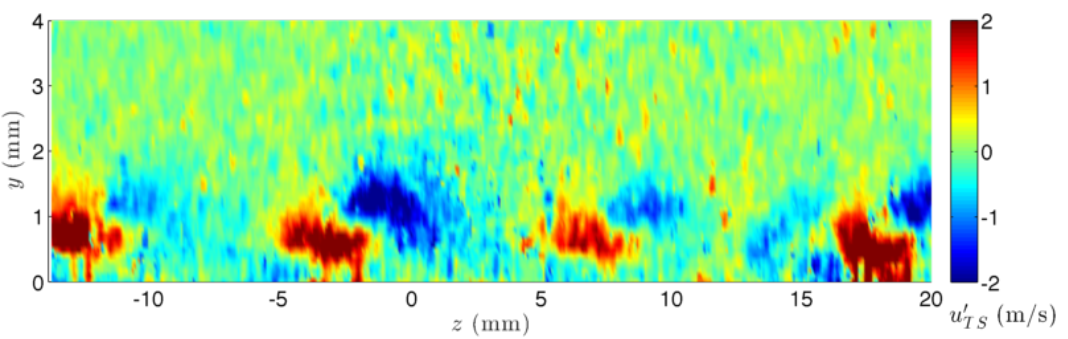

(c) TS

Figure 15: Streamwise velocity, TCF and TS band-pass filtered data during a spike event at $x_{s h}=\mathbf{5 2}$.

The POD analysis is performed individually on the two band-pass-filtered data sets. The first five modes from the POD analysis on the full data set are shown in Fig. 16. At this streamwise location, the instabilities are beginning to undergo a strong spanwise modulation due to the action of the stationary crossflow vortices. All five POD modes of the TS band-pass-filtered data (right column) exhibit a clear $11 \mathrm{~mm}$ spanwise wavelength. The second mode (Fig. 16d) looks similar to the TS snapshot during the spike event (Fig. 15c). The first two modes of the traveling crossflow band-pass-filtered data (Figs. 16a and 16c show some remnant of the large wavelength spanwise traveling mode that was evident at the upstream stations (Fig. 9). The shift in phase between mode one and two indicates that these modes form a pair, physically indicating the presence of a traveling mode in the spanwise plane. The third through fifth modes (Fig. 16e g,i), however, exhibit a clear spanwise wavelength of the dominant stationary crossflow mode $(11 \mathrm{~mm})$. The third mode is similar to the TCF snapshot during the spike event (Fig. 15b). This mode, when superposed onto the mean flow with a positive coefficient, results in an increase in the spanwise modulation of the flow initially caused by the stationary crossflow. This effect is illustrated in Fig. 17, which shows the time-averaged velocity at $y=1$ across the span compared to the time-averaged velocity with the addition of the traveling crossflow POD mode 3 (Fig. 16e) with a large positive and negative coefficient. As this figure illustrates, when this mode is superposed with a positive coefficient, there is an increase in the spanwise modulation compared to the mean flow. However, the opposite is true when a negative coefficient (representing a $180^{\circ}$ phase shift) is applied. 


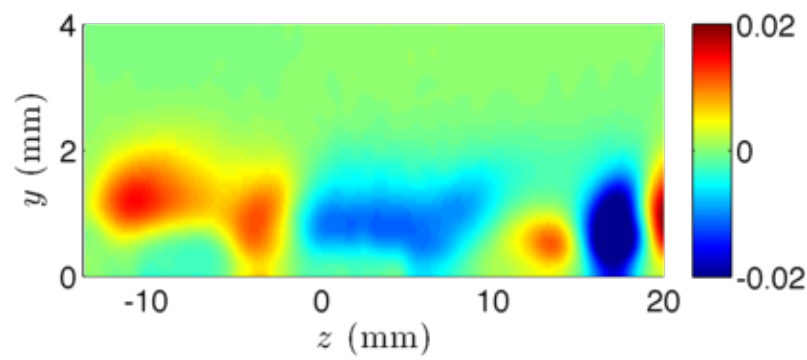

(a) Mode 1, TCF

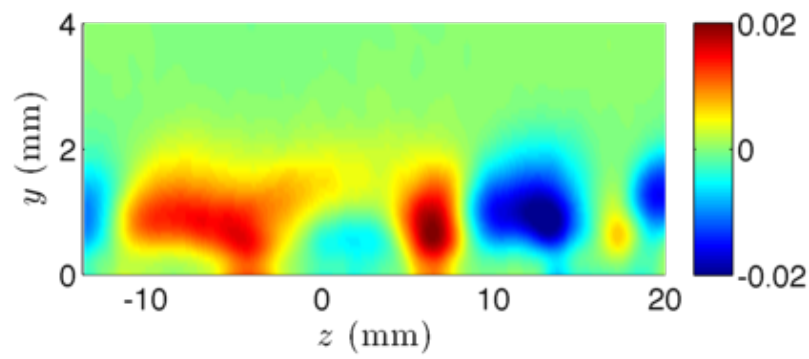

(c) Mode 2, TCF

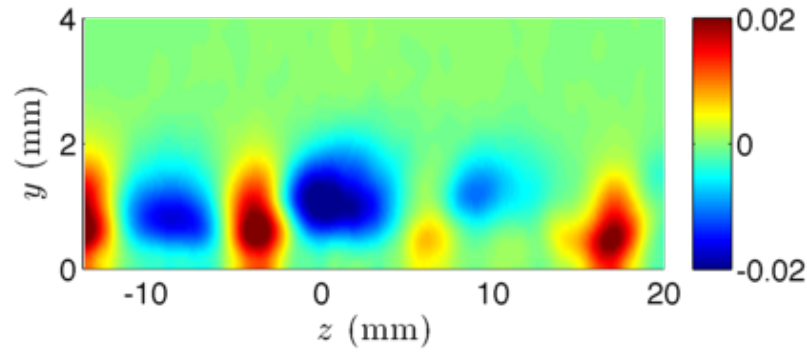

(e) Mode 3, TCF

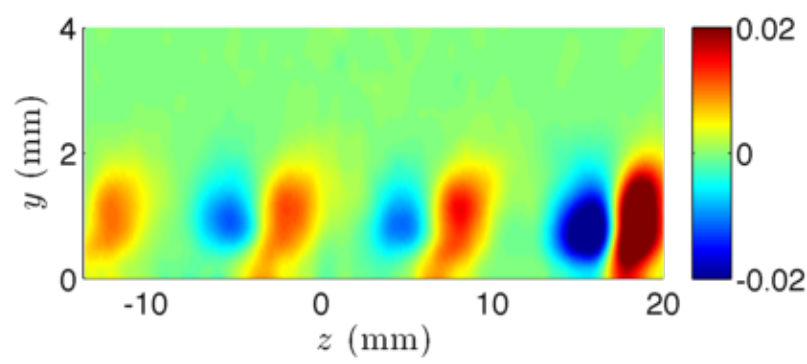

(g) Mode 4, TCF

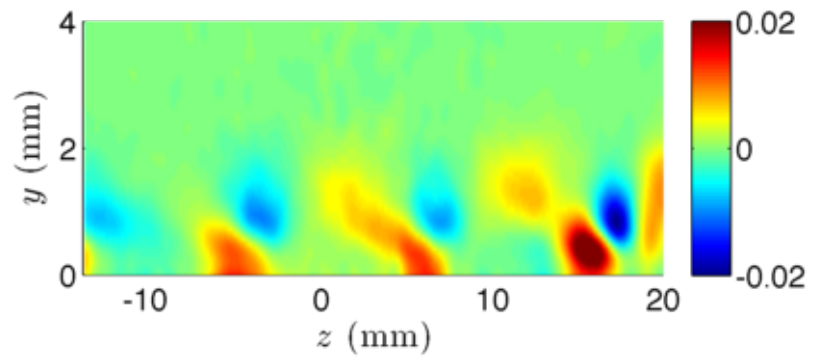

(i) Mode 5, TCF

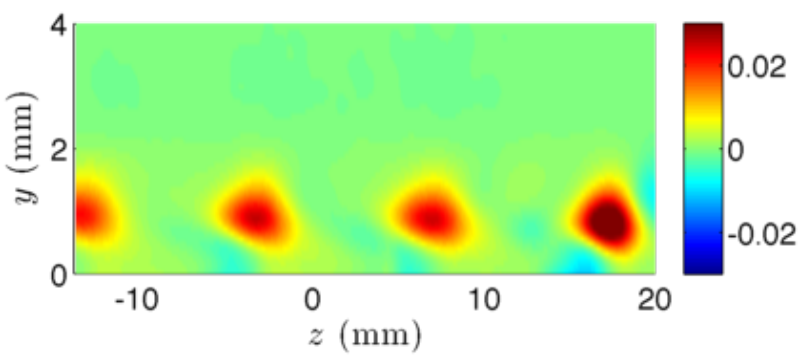

(b) Mode 1, TS

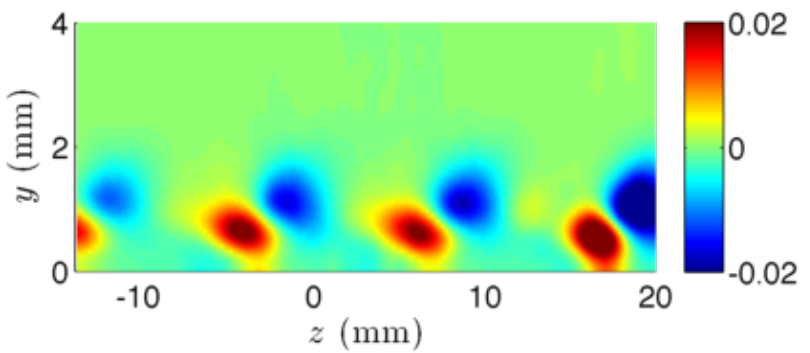

(d) Mode 2, TS

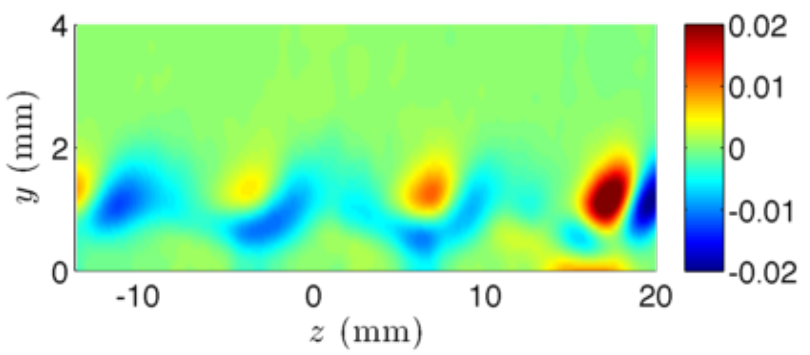

(f) Mode 3, TS

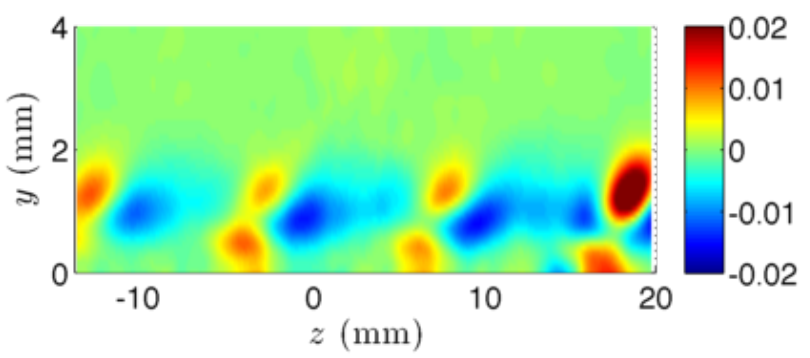

(h) Mode 4, TS

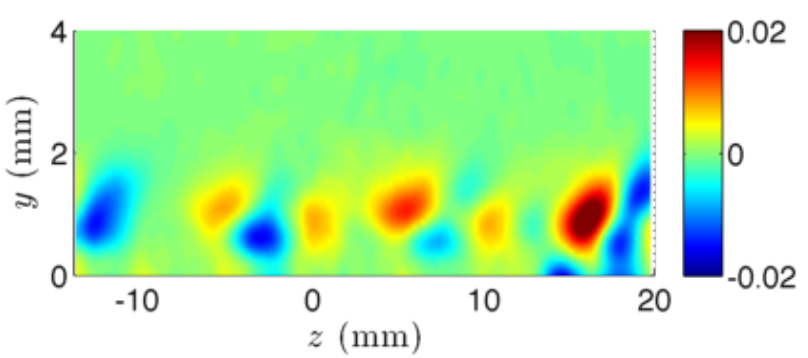

(j) Mode 5, TS

Figure 16: First five POD modes for the traveling crossflow and TS bandpass-filtered full data sets, $x_{s h}=52$. 


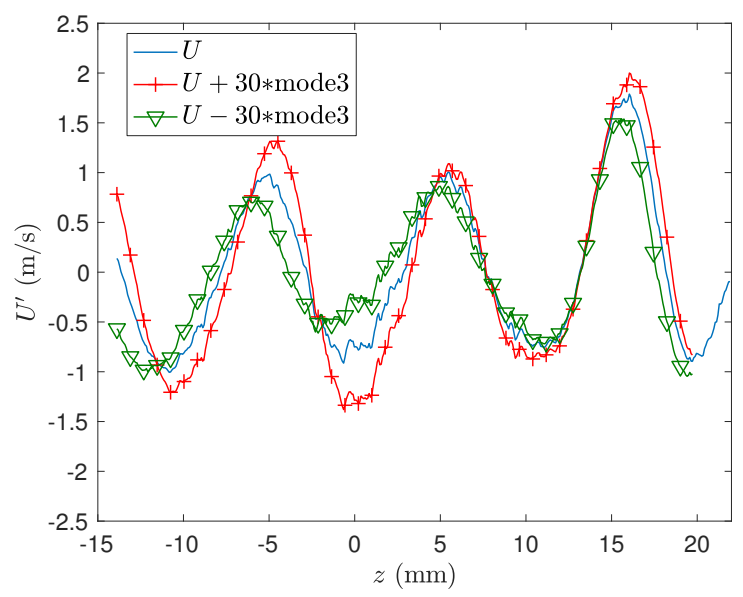

Figure 17: Comparison of spanwise modulation of mean flow with and without TCF POD mode 3 .

We explore the traveling crossflow modes further to try to determine the physical nature of the different sets of POD modes. Since longer wavelength structures are apparent in the first two POD modes, and the 11 $\mathrm{mm}$ wavelength structures are more dominant in modes 3 through 5 , it appears that these two sets of modes may constitute different physical structures. A 0.085 second long stretch of data is reconstructed using just the first two POD modes (Fig. 18a), and then separately the third through fifth POD modes (Fig. 18b). As expected, the reconstruction using the first two modes shows a slightly modulated spanwise-traveling wave with a long wavelength, similar to that seen in the POD mode reconstructions farther upstream (Fig. 11). The reconstruction using modes 3 through 5 illustrates a very different structure that exists in this same frequency band. This structure may also be a spanwise-traveling wave, but with a smaller spanwise wavelength, $\lambda_{z} \approx$ 8.5 to $20 \mathrm{~mm}$, calculated based on the phase speed in the spanwise plane and the frequency band of 80 to $200 \mathrm{~Hz}$. During this particular span of time, the mode primarily appears to be traveling in the outboard (positive $z$ ) direction, though there are times where it changes direction and appears to be traveling in the opposite direction, such as $t=0$ to $0.01 \mathrm{~s}$. It is also possible that this mode is not a spanwise traveling crossflow instability, but instead an oblique wave, or a pair of oblique waves similar to that seen in the TS frequency band starting at $x_{s h}=52$ (see Fig. 12). Perhaps this is another set of oblique TS modes resulting from the TS-stationary crossflow interaction, but at a lower frequency. Unfortunately, we cannot draw any definitive conclusions about what type of instability this is based on the available data.

Next, the POD analysis is applied to the subset of snapshots corresponding to the times when the large velocity spikes occur. These samples are identified by using the time series shown in Fig. 14. A threshold of $u^{\prime}=1.7 \mathrm{~m} / \mathrm{s}$ is chosen to be representative of a spike event (which is twice the standard deviation of $u^{\prime}(t)$ at this location), and the peaks above this threshold are identified from the time series. This results in 108 spike samples over which to perform POD. Note that we have also performed this analysis using a time series from a different location (such as a location where negative spikes occur), and the results are similar since the spikes occur nearly simultaneously across the whole plane of data. The first POD modes for the traveling crossflow and TS frequency bands from this analysis are shown in Fig. 19. These two modes also appear in the POD modes from the full set of data (Fig. 16), but they are not the first modes. The traveling-crossflow first mode from the POD analysis of the spike samples (Fig. 19a) corresponds to the third mode from the POD analysis of the full data set (Fig. 16e). The TS first mode from the POD analysis of the spike samples (Fig. 19b most closely resembles the second mode from the POD analysis of the full data set (Fig. 16d). The POD energy spectra for the first 50 POD modes for both the traveling crossflow and TS results are shown in Fig. 20. The first mode for the TCF data contains over $50 \%$ of the total energy, while the first mode for the TS data contains over $70 \%$ of the total energy. Thus, these modes are clearly very dominant during the spike events. 


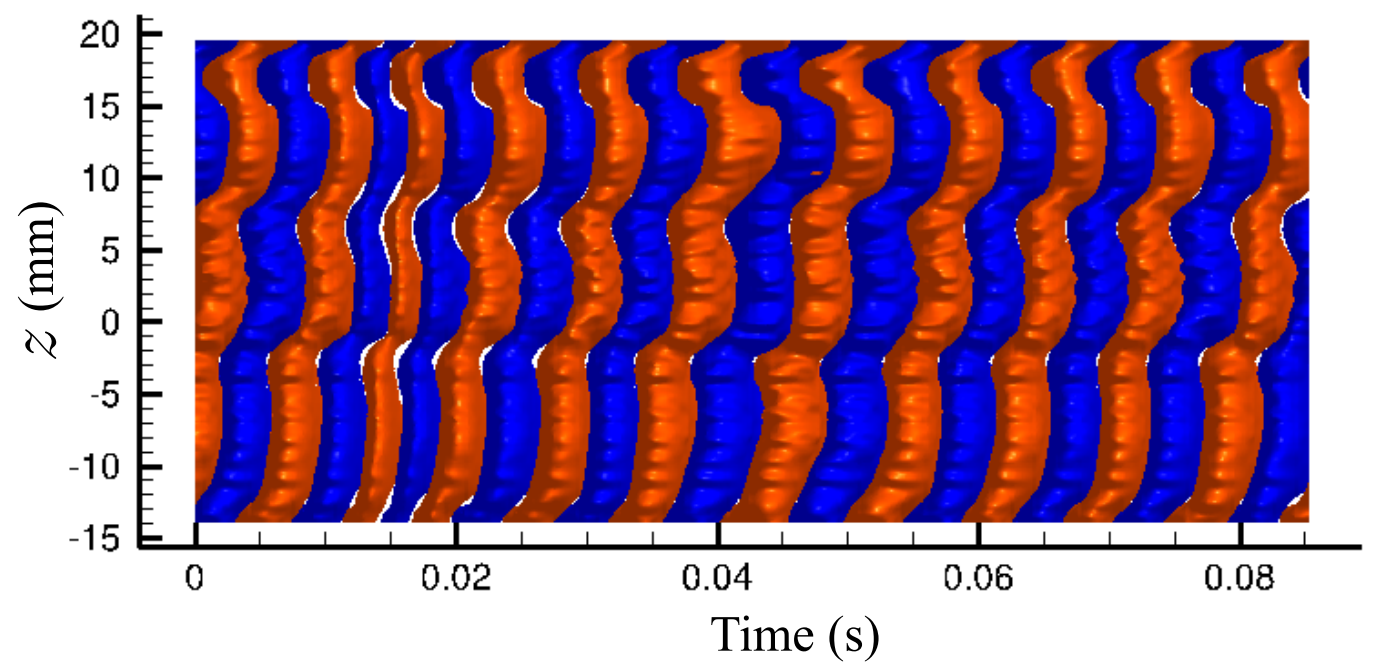

(a) POD modes 1-2

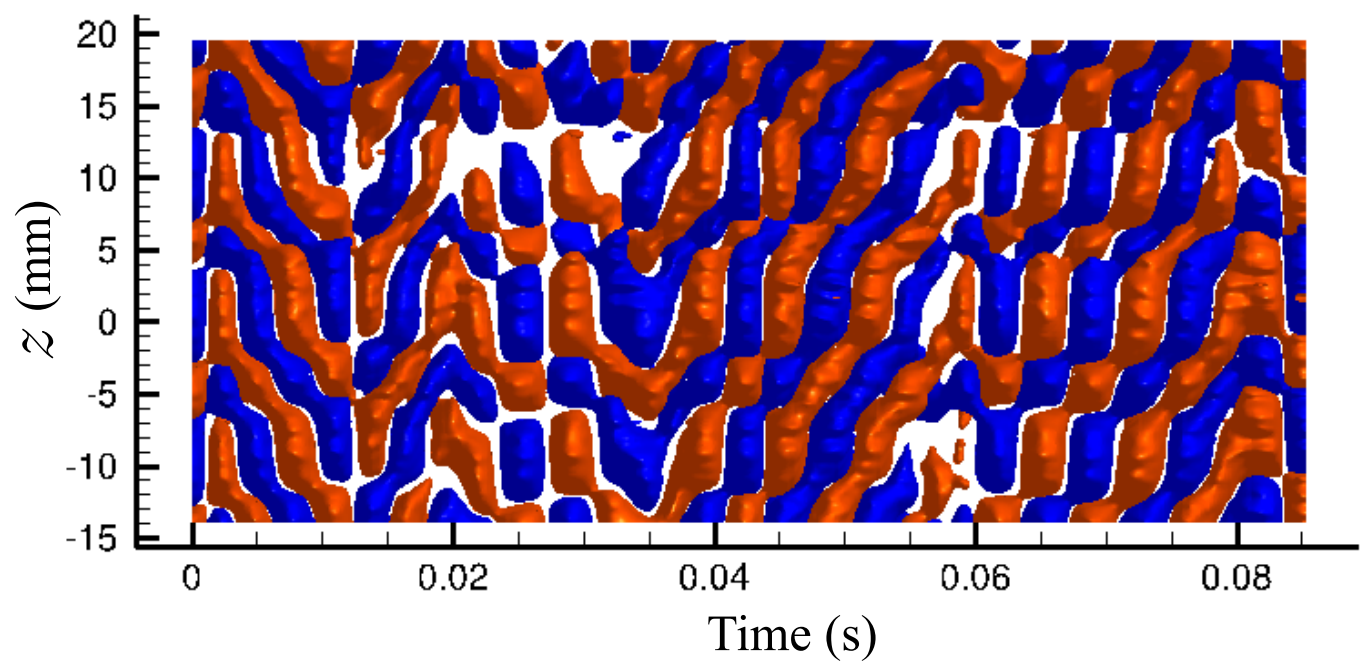

(b) POD modes 3-5

Figure 18: Reconstruction of TCF frequency band using first two POD modes and modes 3 through 5 separately, $x_{s h}=52$.

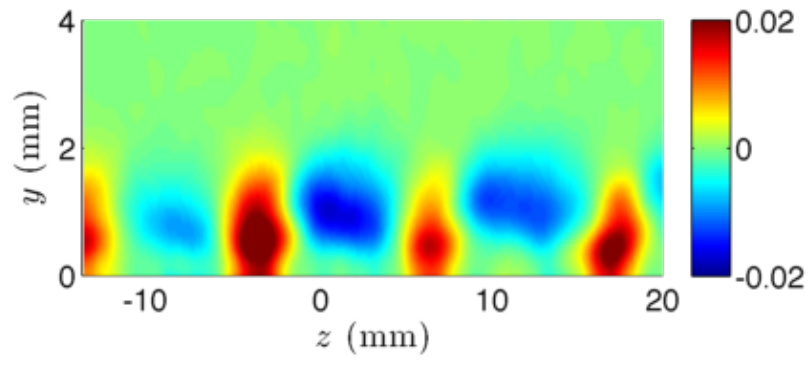

(a) TCF

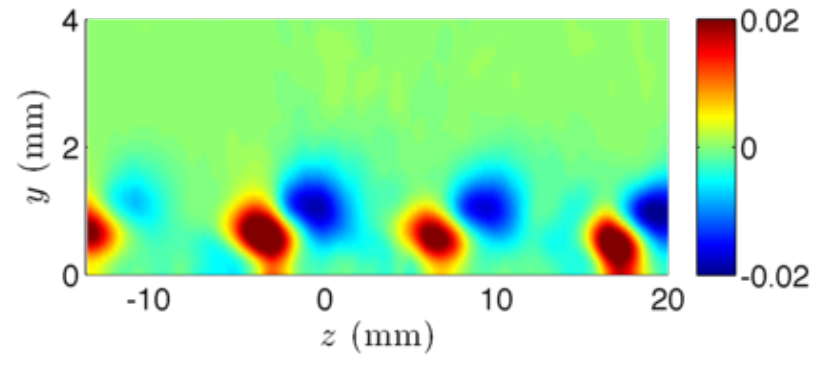

(b) TS

Figure 19: First POD mode from spike subset of data.

To illustrate the effect of the spike events on the entire flow field, we also show the first POD mode from the spike subset of snapshots taken from the unfiltered data set. This first POD mode is shown in Fig. 21a 
and essentially looks like a combination of the TCF and TS first modes shown in Fig. 19, Figure 21b shows a superposition of the time-averaged streamwise velocity (shown in Fig. 13a) with the POD mode applied with a coefficient of 50, showing the effect of this POD mode on the mean flow. The enhanced modulation of this flow field is evident when compared to the mean flow in Fig. 13a and is similar to the effect seen for the single snapshot during the spike event shown in Fig. 15a.

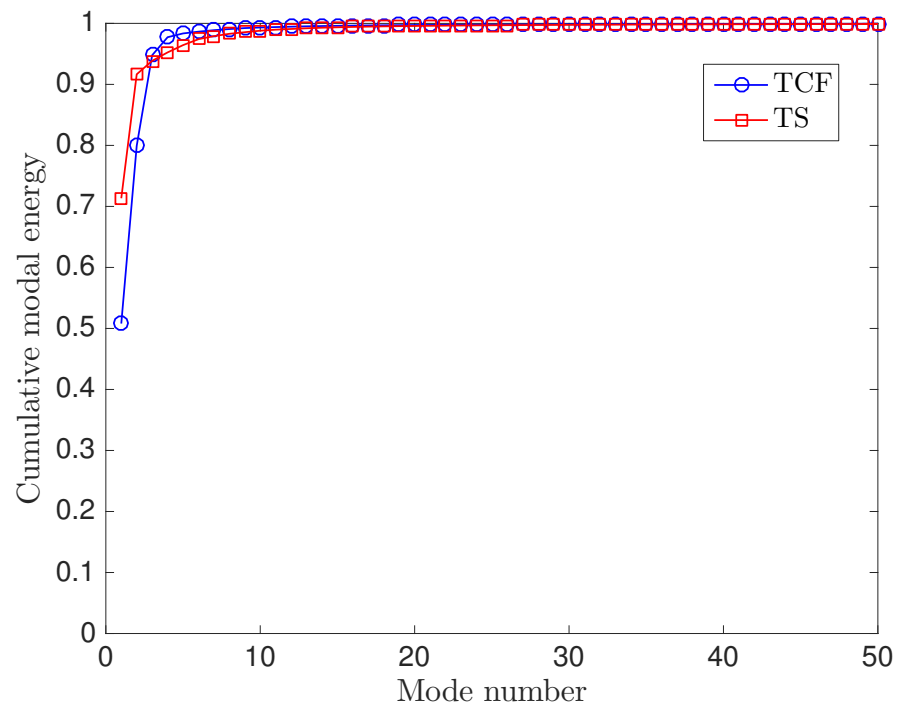

Figure 20: Cumulative modal energy of first 50 POD modes from spike subset of data.

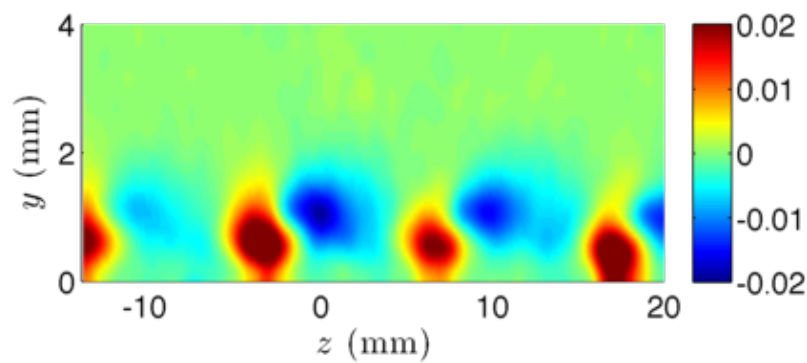

(a) First POD mode from spike subset of unfiltered data.

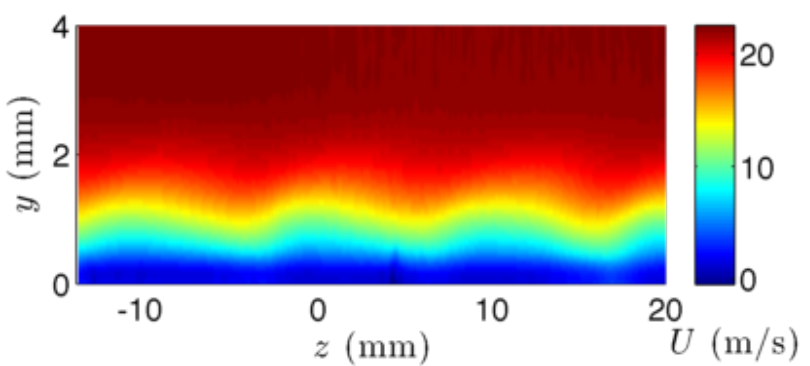

(b) Time-averaged streamwise velocity field plus the first POD mode with a coefficient of 50 .

Figure 21: First POD mode from spike subset of unfiltered data and its effect on the mean flow.

Histograms of the POD coefficients for the first modes from the spike subset of data are shown in Fig. 22. The coefficients are mostly positive for both modes, indicating that the spikes primarily occur during the positive part of the phase (i.e., when they are both positive near the wall, as illustrated in Fig. 19p. Additionally, a scatter plot of the coefficients for the traveling crossflow and TS first modes (Fig. 23) indicates a direct relationship between the amplitude and phase (i.e, sign) of the two modes during the spike events. In context of the full data set, these two modes do not occur more often with a positive phase rather than a negative phase. This can be illustrated by examining the histograms of the POD coefficients of the modes from the full data set that most closely resemble the first modes from the spike subset. Fig 24 shows the histograms of the coefficients for the third mode from the traveling-crossflow-filtered data (Fig. 16e), and the second mode from the TS-filtered data (Fig. 16d). In contrast to the coefficients from the spike subset of data (Fig. 22), these histograms are centered around zero and are not skewed either positively or negatively. This indicates that the positive phase of these modes is correlated with the spike events, but in general the positive and negative phases occur equally. 


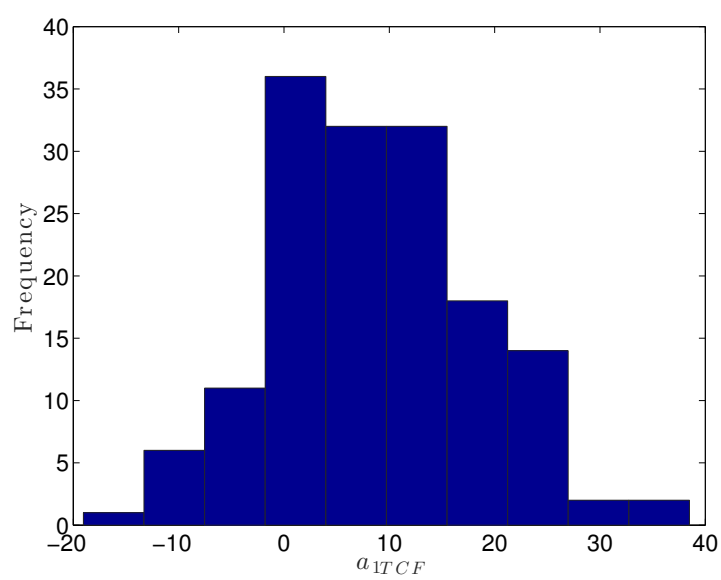

(a) TCF

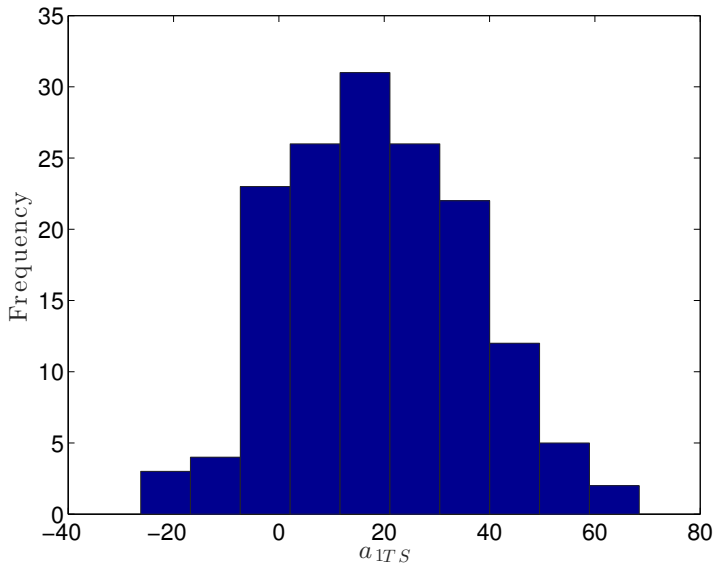

(b) TS

Figure 22: First POD mode coefficients from spike subset of data.

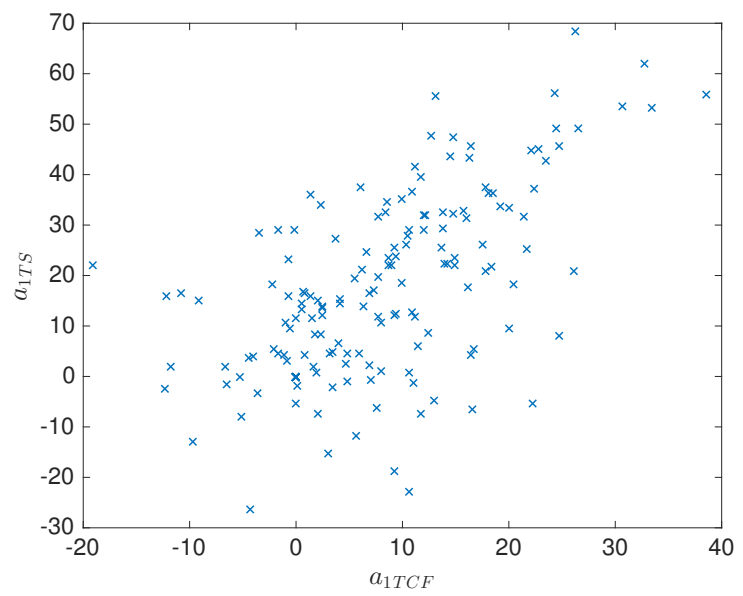

Figure 23: Scatter plot of first mode coefficients from spike subset of data.

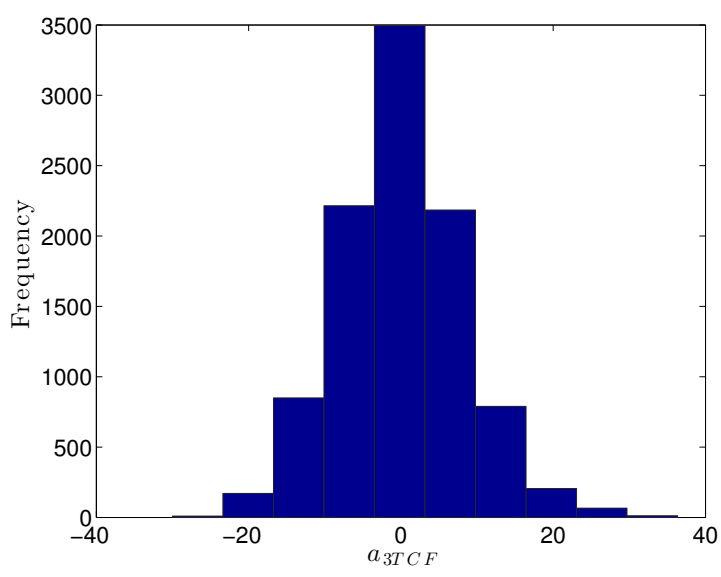

(a) TCF, Mode 3

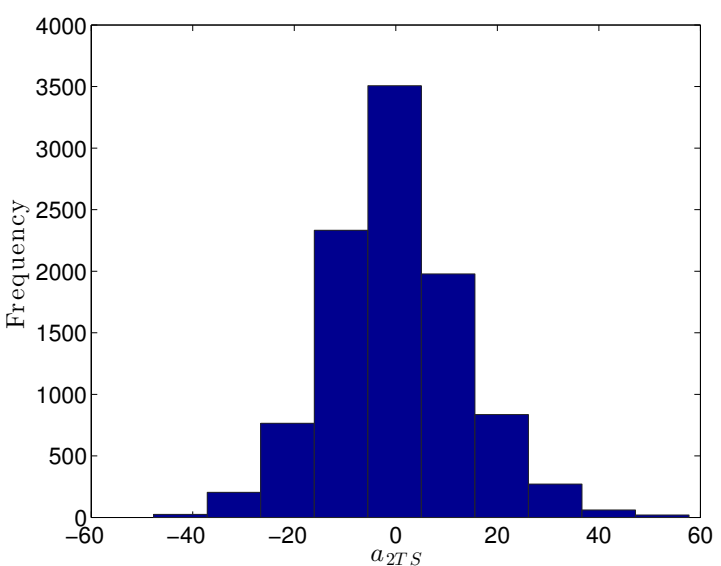

(b) TS, Mode 2

Figure 24: POD mode coefficients from full data set. 
Scatter plots of these POD coefficients are shown in Fig. 25 in order to examine the relationship between these TCF and TS modes. We plot the first POD mode for the TS-filtered data in addition to the second POD mode because these two modes make up the majority of the energy for this instability. In both figures, the $\mathrm{x}$-axis is the coefficient of the third POD mode of the traveling-crossflow filtered data. This value is plotted against the first mode coefficient for the TS filtered data in Fig. 25a, and against the second mode coefficient in Fig. 25b. These plots show an interesting relationship between these two sets of coefficients. Specifically, as the coefficient of the traveling-crossflow mode increases, the scatter of both of the TS POD modes increases (i.e., they reach larger absolute values). To further illustrate this relationship, we divided the TCF mode coefficients into 20 bins and calculated the rms amplitude of the TS POD mode coefficients within each bin. These values, multiplied by $\pm \sqrt{2}$ to illustrate the peak amplitudes, are shown as the red lines in these figures. These lines clearly illustrate the effect of the sign and amplitude of the TCF 3rd POD mode coefficient on the fluctuation amplitude of the two TS POD mode coefficients. This behavior indicates that the amplitude of the TS fluctuations depends on the phase and amplitude of this particular traveling crossflow mode. As was mentioned previously, this traveling crossflow mode causes the modulation of the streamwise velocity to increase during the positive part of the phase. Thus, the enhanced modulation of the flow may be causing an increase in the amplitude of the TS mode.

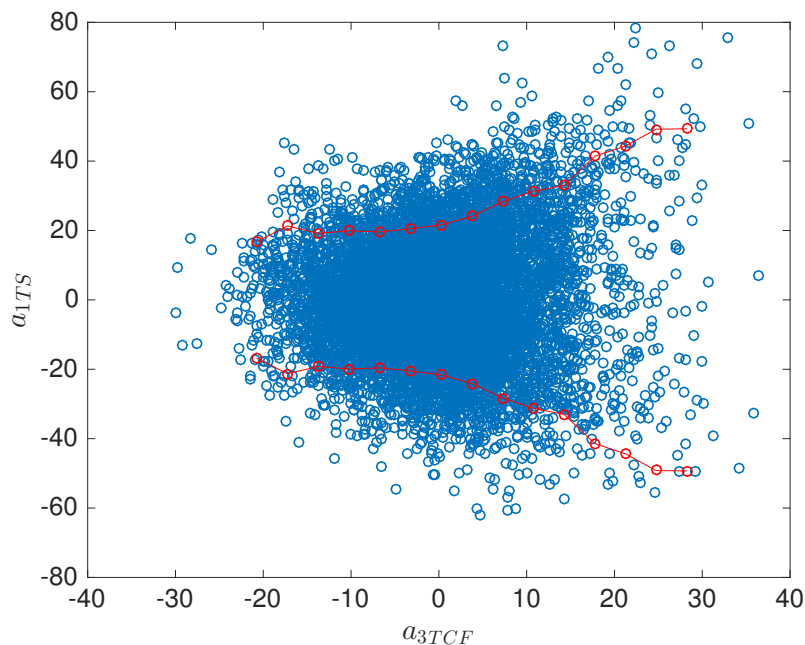

(a) TCF mode 3 vs. TS mode 1 coefficients.

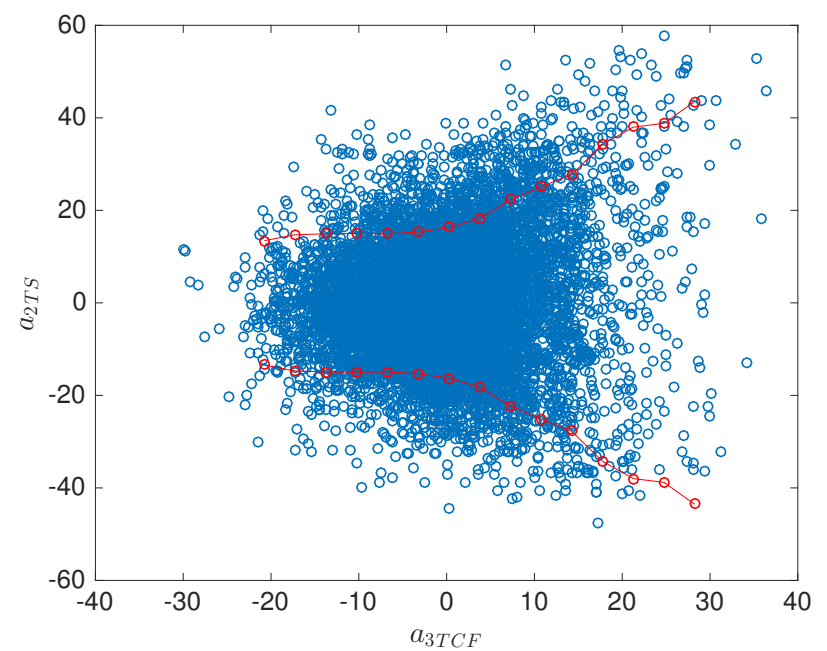

(b) TCF mode 3 vs. TS mode 2 coefficients.

Figure 25: Scatter plot showing relationship between TCF mode 3 coefficients, and TS mode 1 and mode 2 coefficients.

The first POD traveling-crossflow modes at the two downstream stations $\left(x_{s h}=62\right.$ and 73 in Fig. 7) look similar to the third traveling-crossflow POD mode at $x_{s h}=52$ (Fig. 16e). This indicates that this mode becomes more dominant at the downstream locations. Additionally, the large velocity spikes at these stations occur more frequently and are becoming larger in amplitude. By the last station, the flow is starting to become intermittent. The high frequencies typically occur during the spike events, indicating that the breakdown is occurring intermittently as a result of the large flow modulation that occurs during the large velocity spikes. Figure 26a shows a time trace from a location near the wall at $x_{s h}=73$, zoomed in to show the high frequencies occurring during the large velocity spike. The instantaneous streamwise velocity field in the spanwise plane during the spike at $t=0.231$ seconds is shown in Fig. 26b. This flow field, compared to that shown in Fig. 15a at $x_{s h}=52$, is fairly chaotic and reveals the existence of large-amplitude small-wavelength structures, indicating the beginning of breakdown. 


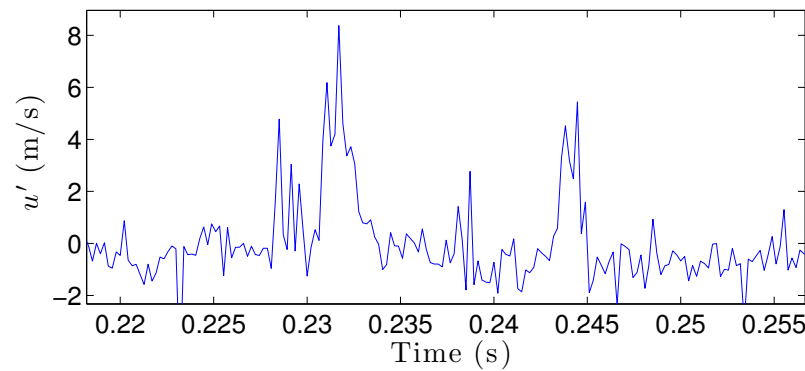

(a) Time trace of $u^{\prime}$ at $z=-3 \mathrm{~mm}, y=0.5 \mathrm{~mm}$.

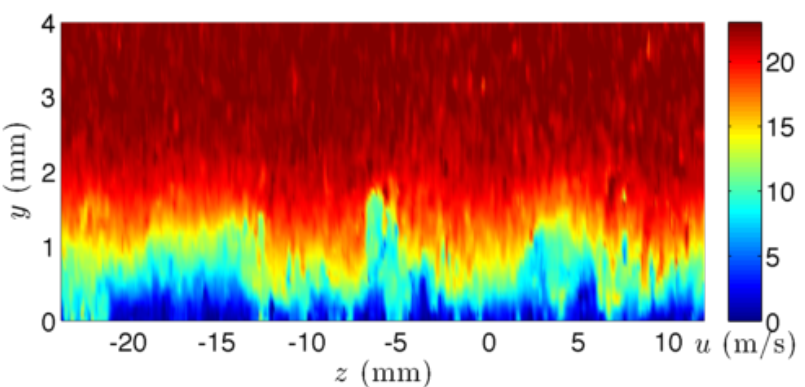

(b) Streamwise velocity field at $t=0.231$ seconds.

Figure 26: Time trace and streamwise velocity field showing spike event, $x_{s h}=73$.

\section{Summary and Conclusions}

Time-resolved PIV measurements of the flow instabilities downstream of a backward-facing step in a swept wing flow are presented. The TRPIV measurements, though much noisier than the previously reported hotwire measurements, were still able to resolve the low-amplitude instabilities in the flow. The TRPIV measurements agree well qualitatively with the hotwire results. The spectra show the same broadband peaks around the traveling crossflow and TS frequency band, and the contours of RMS fluctuating velocity reveal the same modulation patterns that occur due to interactions with the stationary crossflow vortices. The fluctuating amplitudes measured using TRPIV were somewhat higher than the corresponding hotwire amplitudes, which is likely a consequence of the additional noise inherent in the technique. However, it is encouraging that TRPIV was able to resolve the instabilities at such low amplitudes. The results provide valuable insight into the development and interactions of the instabilities that cannot be obtained using a point measurement technique such as hotwire anemometry.

In the previously-reported hotwire measurements, large velocity spikes were observed to occur prior to breakdown downstream of the backward-facing step. These velocity spikes grew larger in amplitude downstream and eventually experienced high-frequency oscillations, indicating the beginning of breakdown. The TRPIV results allow us to study the downstream development of the traveling crossflow and TS instabilities and to see how they interact to produce this particular path to breakdown.

The TS instability becomes highly modulated due to interactions with the stationary crossflow instability. This was known from the hotwire data, but the TRPIV results also reveal more details about the highly three-dimensional nature of this instability downstream of reattachment, as well as the possibility of the existence of a pair of oblique modes starting at $x_{s h}=52$.

The traveling crossflow mode begins as a large wavelength $\left(\lambda_{z} \approx 40 \mathrm{~mm}\right)$ spanwise traveling mode, which was also observed from the hotwire measurements. POD analysis indicates the existence of another mode within this same frequency range beginning downstream of reattachment. At a certain point in the phase, this mode enhances the streamwise velocity modulation initially caused by the stationary crossflow instability. This occurs because the spanwise wavelength of this traveling crossflow mode is similar to that of the primary stationary crossflow mode. It is at this point that the large amplitude velocity spikes tend to occur, but only when the TS mode is also at a similar point in its phase, such that the positive and negative parts of the mode mostly align with the TCF mode, further enhancing the modulation of the flow. However, the velocity spikes are not simply a superposition of the two modes. This is evident from the fact that the spikes do not occur during the opposite part of the phases $\left(180^{\circ}\right.$ phase shift $)$ of the TCF and TS modes. The POD analysis also indicates that the enhanced modulation of the flow caused by this TCF mode results in an increase in the fluctuation amplitude of the TS mode. Thus, the large velocity spikes appear to be a result of multiple interactions. First, the stationary crossflow instability causes modulations of the TCF and TS modes. The TCF mode, at the right point in the phase, causes an instantaneous increase in the spanwise flow modulation, which results in an increase in amplitude of the TS mode. The TS mode and the TCF mode then combine, at the right point in the phase of both disturbances, to cause a large spike in velocity. These large spikes cause a very large modulation of the flow, which ultimately results in intermittent breakdown.

These results highlight the mechanism through which the instabilities in a low-amplitude broadband disturbance field in a three-dimensional flow can interact to cause large amplitude disturbances, and ulti- 
mately, breakdown and transition. The fluctuating instabilities, whether initially 2D or 3D, become highly modulated through interactions with the stationary crossflow vortices, resulting in points in the phase where they increase the spanwise shear of the flow at certain locations. This further destabilizes the instabilities at these locations, leading to even larger modulations of the flow, eventually resulting in the large amplitude velocity spikes when the multiple types of instabilities are superposed at these locations. In this scenario, the stationary crossflow instabilities, though not strongly impacted by the backward-facing step, play an important role in transition through the modulation of the mean flow and the unsteady instabilities.

\section{References}

${ }^{1}$ Perraud, J. and Seraudie, A., "Effects of Steps and Gaps on 2D and 3D Transition," European Congress on Comp. Methods in Applied Science and Eng., ECCOMAS, 2000, pp. 11-14.

${ }^{2}$ Duncan Jr, G., Crawford, B., and Saric, W., "Effects of Step Excrescences on Swept-Wing Transition," AIAA Paper 2013-2412, 2013.

${ }^{3}$ Eppink, J., Wlezien, R., King, R., and Choudhari, M., "The Interaction of Stationary Crossflow and a Backward-facing Step in Swept Boundary-Layer Transition," AIAA Paper 2015-0273, 2015.

${ }^{4}$ Tufts, M. W., Reed, H. L., Crawford, B. K., Duncan Jr, G. T., and Saric, W. S., "Computational Investigation of Step Excrescence Sensitivity in a Swept-Wing Boundary Layer," Journal of Aircraft, 2016.

${ }^{5}$ Duncan Jr, G. T., Crawford, B. K., Tufts, M. W., Saric, W. S., and Reed, H. L., "Effects of Step Excrescences on a Swept Wing in a Low-Disturbance Wind Tunnel," AIAA Paper 2014-0910, 2014.

${ }^{6}$ Saeed, T. I., Mughal, M. S., and Morrison, J., "The Interaction of a Swept-Wing Boundary Layer with Surface Excrescences," AIAA Paper 2016-2065, 2016.

${ }^{7}$ Eppink, J. and Yao, C.-S., "Stereo Particle Image Velocimetry Measurements of Transition Downstream of a BackwardFacing Step in a Swept-Wing Boundary Layer," AIAA Paper 2017-0306, 2017.

${ }^{8}$ Saric, W. S. and Reshotko, E., "Review of Flow Quality Issues in Wind Tunnel Testing," AIAA Paper 1998-2613, 1998.

${ }^{9}$ Eppink, J., The Interaction of Crossflow Instabilities and a Backward-Facing Step in Swept Boundary Layer Transition, Ph.D. thesis, Tufts University, 2014.

${ }^{10}$ Lumley, J. L., "The Structure of Inhomogeneous Turbulent Flows," Atmospheric Turbulence and Radio Wave Propagation, Vol. 790, 1967, pp. 166-178.

${ }^{11}$ Mei, R., "Velocity Fidelity of Flow Tracer Particles," Experiments in Fluids, Vol. 22, No. 1, 1996, pp. 1-13.

${ }^{12}$ Wassermann, P. and Kloker, M., "Transition Mechanisms in a Three-Dimensional Boundary-Layer Flow with PressureGradient Changeover," Journal of Fluid Mechanics, Vol. 530, 2005, pp. 265-293.

${ }^{13}$ Eppink, J. L., Shishkov, O., Wlezien, R. W., King, R. A., and Choudhari, M. M., "The Effect of Acoustic Forcing on Instabilities and Breakdown in Swept-Wing Flow Over a Backward-Facing Step," AIAA Paper 2016-2063, 2016. 